Article

\title{
Approximation of GBS Type $q$-Jakimovski-Leviatan-Beta Integral Operators in Bögel Space
}

\author{
Abdullah Alotaibi
}

check for updates

Citation: Alotaibi, A. Approximation of GBS Type $q$-Jakimovski-LeviatanBeta Integral Operators in Bögel Space. Mathematics 2022, 10, 675. https://doi.org/10.3390/math 10050675

Academic Editor: Simeon Reich

Received: 25 January 2022

Accepted: 17 February 2022

Published: 22 February 2022

Publisher's Note: MDPI stays neutral with regard to jurisdictional claims in published maps and institutional affiliations.

Copyright: (C) 2022 by the authors. Licensee MDPI, Basel, Switzerland. This article is an open access article distributed under the terms and conditions of the Creative Commons Attribution (CC BY) license (https:// creativecommons.org/licenses/by/ $4.0 /)$.
Operator Theory and Applications Research Group, Department of Mathematics, Faculty of Science, King Abdulaziz University, Jeddah 21589, Saudi Arabia; mathker11@hotmail.com

Abstract: In the present article, we introduce the bivariate variant of Beta integral type operators based on Appell polynomials via $q$-calculus. We study the local and global type approximation properties for these new operators. Next, we introduce the GBS form for these new operators and then study the degree of approximation by means of modulus of smoothness, mixed modulus of smoothness and Lipschitz class of Bögel continuous functions.

Keywords: Szász operators; q-integers; Appell polynomial; Jakimovski-Leviatan operators; simultaneous approximation; mixed-modulus of continuity; Bögel functions

MSC: 41A25; 41A36; 33C45

\section{Introduction and Preliminaries}

In 1950, very famous mathematician, Szász, introduced the operators known as Szász positive linear operators [1]. Suppose $v \in[0, \infty)$ and the class of all continuous functions on $[0, \infty)$ is $C[0, \infty)$, then, for all $f \in C[0, \infty)$, the Szász operators are defined as:

$$
S_{r}(f ; v)=e^{-r v} \sum_{k=0}^{\infty} \frac{(r v)^{k}}{k !} f\left(\frac{k}{r}\right) .
$$

Later, the development of Szász operators introduced by the identity of Appell polynomials [2] and an advance technique of Appell polynomials were been addressed by Jakimovski and Leviatan in 1969 [3] by:

$$
X(v) e^{v x}=\sum_{k=0}^{\infty} \beta_{k}(x) v^{k}
$$

where $\beta_{k}(x)=\sum_{i=0}^{k} \alpha_{i} \frac{x^{m-i}}{(m-i) !}$ for $m \in \mathbb{N}$ and $X(v)=\sum_{k=0}^{\infty} \alpha_{k} v^{k}, X(1) \neq 0$. Let $E[0, \infty)$ denote the set of functions defined by $[0, \infty)$ such that $|f(x)| \leq \kappa e^{\gamma x}$, where $\kappa, \gamma$ are positive constants. For further development, we prefer to see the Jakimovski-Leviatan types and other related articles [4-10].

The family of $q$-Appell polynomials introduced by Al-Salam (see $[11,12])$ by using the generating functions $X_{q}(t)=\sum_{m=0}^{\infty} X_{m, q} \frac{t^{m}}{[m]_{q} !}, X_{q}(1) \neq 0$, such that:

$$
X_{m, q}(x)=\sum_{k=0}^{m}\left[\begin{array}{c}
m \\
k
\end{array}\right]_{q} A_{m-k, q} x^{k},(m \in \mathbb{N}),
$$

where the $q$-differential $D_{q, x}\left(X_{m, q}(x)\right)=[m]_{q} X_{m-1, q}(x), m=1,2, \ldots$ and notice that $D_{q, x}\left(X_{1, q}(x)\right)=[1]_{q} X_{0, q}(x)=X_{0, q}$, with $X_{0, q}$ is a positive constant. In addition, the 
generating function satisfies the equality $X_{q}(t) e_{q}(t x)=\sum_{m=0}^{\infty} X_{m, q}(x) \frac{t^{m}}{[m]_{q} !}, 0<q<1$. Here, we note some basic formulae of $q$-calculus, thus, the $q$-integer is given by:

$$
[\eta]_{q}=\left\{\begin{array}{ll}
\frac{1-q^{\eta}}{1-q}, & q \neq 1 \\
\eta, & q=1
\end{array} \text { for } \eta \in \mathbb{N} \text { and }[0]_{q}=0\right.
$$

For $|q|<1$, the $q$-factorial $[\eta]_{q}$ ! is defined by:

$$
[\eta]_{q} != \begin{cases}1, & (\eta=0) \\ \prod_{k=1}^{\eta}[k]_{q^{\prime}} & (\eta \in \mathbb{N}) .\end{cases}
$$

In the standard approach, the exponential functions for $q$-calculus:

$$
\begin{gathered}
e_{q}(x)=\sum_{k=0}^{\infty} \frac{x^{k}}{[k]_{q} !^{\prime}} \\
E_{q}(x)=\sum_{k=0}^{\infty} \frac{q^{\frac{k(k-1)}{2}} x^{k}}{[k]_{q} !},
\end{gathered}
$$

while the $q$-Jackson improper integral of the function $f$ is given as:

$$
\int_{0}^{\infty / A} f(x) d_{q} x=(1-q) \sum_{m \in \mathbb{N}} f\left(\frac{q^{m}}{A}\right) \frac{q^{m}}{A}, A \in \mathbb{R}-\{0\},
$$

where $\mathbb{R}$ is the set of real numbers.

The $q$-beta function $B_{q}(l, m)$ is defined as follows:

$$
B_{q}(l, m) \int_{0}^{1} t^{l-1}(1-q t)_{q}^{m-1} d_{q} t \quad(l>0 ; m>0)
$$

and for $l>1 ; m>0$

$$
B_{q}(l, m)=\mathcal{K}(A, l) \int_{0}^{\infty / A} \frac{t^{l-1}}{(1+t)_{q}^{l+m}} d_{q} t=\frac{[l-1]_{q}}{[m]_{q}} B_{q}(l-1, m+1)
$$

with the explicit form of:

$$
\begin{gathered}
\mathcal{K}(A, l+1)=q^{l} \mathcal{K}(A, l) . \\
\mathcal{K}(A, l)=\frac{1}{A+1} A^{l}\left(1+\frac{1}{A}\right)_{q}^{l}(1+A)_{q}^{1-l},
\end{gathered}
$$

while the Gamma function in the $q$ - form is defined as follows:

$$
\Gamma_{q}(x)=\int_{0}^{1 / 1-q} t^{x-1} E_{q}(-q t) d_{q} t \quad(x>0)
$$

satisfying $\Gamma_{q}(x+1)=[x]_{q} \Gamma_{q}(x), \Gamma_{q}(1)=1$, and

$$
\Gamma_{q}(x)=\int_{0}^{\infty / 1-q} t^{x-1} E_{q}(-q t) d_{q} t \quad(x>0)
$$


and a relation of the Beta and Gamma functions given as:

$$
B_{q}(l, m)=\frac{\Gamma_{q}(l) \Gamma_{q}(m)}{\Gamma_{q}(l+m)} .
$$

In the present context, we consider the recent development of the published article [13] and then construct the operators in terms of two variables supposing $0 \leq v_{1}, v_{2}<\infty$. Finally, we study the approximation results in terms bivariate functions by modulus and mixed-modulus of continuity and Lipschitz maximal functions. Next, we also construct the GBS-type polynomial functions and obtain the approximation in terms of Bögel continuous functions. For more related concepts on these classes of functions, we prefer to see the recent published article by Nasiruzzaman et al. [14-19]. In addition, there are various operators in several functional spaces given by the authors: Mohiuddine et al. [20-23], Mursaleen et al. [24,25], Acar et al. [26], Kajla et al. [27], Özger et al. [28], and Rao et al. [29,30].

\section{Operators and Their Associated Moments}

Take $\mathcal{M}^{2}=\left\{\left(v_{1}, v_{2}\right): 0 \leq v_{1}<\infty, 0 \leq v_{2}<\infty\right\}$, and $C\left(\mathcal{M}^{2}\right)$ is the class of all continuous functions on $\mathcal{M}^{2}$ and satisfies the norm by $\|g\|_{C\left(\mathcal{M}^{2}\right)}=\sup _{\left(v_{1}, v_{2}\right) \in \mathcal{M}^{2}}\left|f\left(v_{1}, v_{2}\right)\right|$. Suppose $f \in C\left(\mathcal{M}^{2}\right)$. Then, for all $i=1,2$ such that $m_{i} \in \mathbb{N}, X_{r_{i}, q_{i}}\left(\left[m_{i}\right]_{q_{i}} v_{i}\right) \geq 0$ and $X_{q_{i}}(1) \neq 0$, we define:

$$
\mathcal{J}_{m_{1}, m_{2}}^{q_{1}, q_{2}}\left(f ; v_{1}, v_{2}\right)=\sum_{r_{1}, r_{2}=0}^{\infty} \mathcal{U}_{m_{1}, q_{1}}^{r_{1}}\left(v_{1}\right) \mathcal{V}_{m_{2}, q_{2}}^{r_{2}}\left(v_{2}\right) \int_{0}^{\infty / A_{1}} \int_{0}^{\infty / A_{2}} \mathcal{T}_{m_{1}, m_{2}}^{q_{1}, q_{2}}(t, s) f\left(q_{1}^{r_{1}} t, q_{2}^{r_{2}} s\right) d_{q_{1}} t d_{q_{2} s} s,
$$

where

$$
\begin{aligned}
& \mathcal{U}_{m_{1}, r_{1}}^{q_{1}}\left(v_{1}\right)=\frac{e_{q_{1}}\left(-\left[m_{1}\right]_{q_{1}} v_{1}\right)}{X_{q_{1}}(1)} \frac{R_{r_{1}, q_{1}}\left(\left[m_{1}\right]_{q_{1}} v_{1}\right)}{\left[r_{1}\right]_{q_{1}} !} \frac{\mathcal{K}_{1}\left(A_{1}, r_{1}+1\right)}{B_{q_{1}}\left(r_{1}+1, m_{1}\right)}, \\
& \mathcal{V}_{m_{2}, r_{2}}^{q_{2}}\left(v_{2}\right)=\frac{e_{q_{2}}\left(-\left[m_{2}\right]_{q_{2}} v_{2}\right)}{X_{q_{2}}(1)} \frac{R_{r_{2}, q_{2}}\left(\left[m_{2}\right]_{q_{2}} v_{2}\right)}{\left[r_{2}\right]_{q_{2}} !} \frac{\mathcal{K}_{2}\left(A_{2}, r_{2}+1\right)}{B_{q_{2}}\left(r_{2}+1, m_{2}\right)}
\end{aligned}
$$

and

$$
\mathcal{T}_{m_{1}, m_{2}}^{q_{1}, q_{2}}(t, s)=\frac{t^{r_{1}}}{(1+t)_{q_{1}}^{r_{1}+m_{1}+1}} \frac{s^{r_{2}}}{(1+s)_{q_{2}}^{r_{2}+m_{2}+1}}
$$

It is noticed that for all $i=1,2$, and $r_{i}>1, m_{i}>0$ we have:

$$
B_{q_{i}}\left(r_{i}, m_{i}\right)=\mathcal{K}_{i}\left(A_{i}, r_{i}\right) \int_{0}^{\infty / A_{i}} \frac{u^{r_{i}-1}}{\left(1+u_{i}\right)_{q_{i}}^{r_{i}+m_{i}}} d_{q_{i}} u_{i}=\frac{\left[r_{i}-1\right]_{q_{i}}}{\left[m_{i}\right]_{q_{i}}} B_{q_{i}}\left(r_{i}-1, m_{i}+1\right),
$$

with

$$
\begin{gathered}
\mathcal{K}_{i}\left(A_{i}, r_{i}+1\right)=q_{i}^{r_{i}} \mathcal{K}_{i}\left(A_{i}, r_{i}\right), \\
\mathcal{K}_{i}\left(A_{i}, r_{i}\right)=q_{i}^{\frac{r_{i}\left(r_{i}-1\right)}{2}}, \mathcal{K}_{i}\left(A_{i}, 0\right)=1 .
\end{gathered}
$$

Lemma 1. Operators $J_{m_{1}, m_{2}}^{q_{1}, q_{2}}\left(f ; v_{1}, v_{2}\right)$ defined by (15) have the equality:

$$
J_{m_{1}, m_{2}}^{q_{1}, q_{2}}\left(f ; v_{1}, v_{2}\right)=\mathcal{C}_{m_{1}, r_{1}}^{q_{1}}\left(\mathcal{D}_{m_{2}, r_{2}}^{q_{2}}\left(f ; v_{1}, v_{2}\right)\right)=\mathcal{D}_{m_{2}, r_{2}}^{q_{2}}\left(\mathcal{C}_{m_{1}, r_{1}}^{q_{1}}\left(f ; v_{1}, v_{2}\right)\right)
$$

where

$$
\begin{gathered}
\mathcal{C}_{m_{1}, r_{1}}^{q_{1}}\left(f ; v_{1}, v_{2}\right)=\sum_{r_{1}=0}^{\infty} \mathcal{U}_{m_{1}, r_{1}}^{q_{1}}\left(v_{1}\right) \int_{0}^{\infty / A_{1}} \frac{t^{r_{1}}}{(1+t)_{q_{1}}^{r_{1}+m_{1}+1}} f\left(q_{1}^{r_{1}} t, q_{2}^{r_{2}} s\right) d_{q_{1}} t \\
\mathcal{D}_{m_{2}, r_{2}}^{q_{2}}\left(f ; v_{1}, v_{2}\right)=\sum_{r_{2}=0}^{\infty} \mathcal{V}_{m_{2}, r_{2}}^{q_{2}}\left(v_{2}\right) \int_{0}^{\infty / A_{2}} \frac{s^{r_{2}}}{(1+s)_{q_{2}}^{r_{2}+m_{2}+1}} f\left(q_{1}^{r_{1}} t, q_{2}^{r_{2}} s\right) d_{q_{2}} s .
\end{gathered}
$$


Proof. We easily see that:

$$
\begin{aligned}
\mathcal{C}_{m_{1}, r_{1}}^{q_{1}}\left(\mathcal{D}_{m_{2}, r_{2}}^{q_{2}}\left(f ; v_{1}, v_{2}\right)\right) & =\mathcal{C}_{m_{1}, r_{1}}^{q_{1}}\left(\sum_{r_{2}=0}^{\infty} \mathcal{V}_{m_{2}, r_{2}}^{q_{2}}\left(v_{2}\right) \int_{0}^{\infty / A_{2}} \frac{s^{r_{2}}}{(1+s)_{q_{2}}^{r_{2}+m_{2}+1}} f\left(q_{1}^{r_{1}} t, q_{2}^{r_{2}} s\right) d_{q_{2}} s\right) \\
& =\sum_{r_{2}=0}^{\infty} \mathcal{V}_{m_{2}, r_{2}}^{q_{2}}\left(v_{2}\right) \mathcal{C}_{m_{1}, r_{1}}^{q_{1}}\left(\int_{0}^{\infty / A_{2}} \frac{s^{r_{2}}}{(1+s)_{q_{2}}^{r_{2}+m_{2}+1}} f\left(q_{1}^{r_{1}} t, q_{2}^{r_{2}} s\right)\right) d_{q_{2}} s \\
& =\sum_{r_{1}, r_{2}=0}^{\infty} \mathcal{U}_{m_{1}, q_{1}}^{r_{1}}\left(v_{1}\right) \mathcal{V}_{m_{2}, q_{2}}^{r_{2}}\left(v_{2}\right) \int_{0}^{\infty / A_{1}} \int_{0}^{\infty / A_{2}} \mathcal{T}_{m_{1}, m_{2}}^{q_{1}, q_{2}}(t, s) f\left(q_{1}^{r_{1}} t, q_{2}^{r_{2}} s\right) d_{q_{1}} t d_{q_{2}} s \\
& =J_{m_{1}, m_{2}}^{q_{1}, q_{2}}\left(f ; v_{1}, v_{2}\right) .
\end{aligned}
$$

On the other hand, we obtain $\mathcal{D}_{m_{2}, r_{2}}^{q_{2}}\left(\mathcal{C}_{m_{1}, r_{1}}^{q_{1}}\left(f ; v_{1}, v_{2}\right)\right)=J_{m_{1}, m_{2}}^{q_{1}, q_{2}}\left(f ; v_{1}, v_{2}\right)$.

Lemma 2. For all $i=1,2$, if we take $R_{r_{i}, q_{i}}\left(\left[m_{i}\right]_{q_{i}} v_{i}\right) \geq 0$ and $X_{q_{i}}(1) \neq 0$, then for each $m_{i} \in \mathbb{N}$, we have:

$$
\begin{aligned}
& \sum_{r_{i}=0}^{\infty} \frac{X_{r_{i}, q_{i}}\left(\left[m_{1}\right] q_{q_{i}} v_{i}\right)}{\left[r_{i}\right]_{q_{i}} !}=X_{q_{i}}(1) e_{q_{i}}\left(\left[m_{i}\right]_{q_{i}} v_{i}\right) \\
& \sum_{r_{i}=0}^{\infty} r_{i} \frac{X_{r_{i}, q_{i}}\left(\left[m_{i}\right]_{q_{i}} v_{i}\right)}{\left[r_{i}\right]_{q_{i}} !}=\left[\left[m_{i}\right]_{q_{i}} X_{q_{i}}(1) v_{i}+X_{q_{i}}^{\prime}(1)\right] e_{q_{i}}\left(\left[m_{i}\right]_{q_{i}} v_{i}\right) \\
& \sum_{r_{i}=0}^{\infty} r_{i}^{2} \frac{X_{r_{i}, q_{i}}\left(\left[m_{i}\right]_{q_{i}} v_{i}\right)}{\left[r_{i}\right]_{q_{i}} !}=\left[\left[m_{i}\right]_{q_{i}}^{2} X_{q_{i}}(1) v_{i}^{2}+2\left[m_{i}\right]_{q_{i}} X_{q_{i}}^{\prime}(1) v_{i}+X_{q_{i}}^{\prime \prime}(1)\right] e_{q_{i}}\left(\left[m_{i}\right]_{q_{i}} v_{i}\right) \\
& \sum_{r_{i}=0}^{\infty} r_{i}^{3} \frac{X_{r_{i}, q_{i}}\left(\left[m_{i}\right]_{q_{i}} v_{i}\right)}{\left[r_{i}\right]_{q_{i}} !}=\left[\left[m_{i}\right]_{q_{i}}^{3} X_{q_{i}}(1) v_{i}^{3}+3\left[m_{i}\right]_{q_{i}}^{2} X_{q_{i}}^{\prime}(1) v_{i}^{2}+3\left[m_{i}\right]_{q_{i}} X_{q_{i}}^{\prime \prime}(1) v_{i}+X_{q_{i}}^{\prime \prime \prime}(1)\right] e_{q_{i}}\left(\left[m_{i}\right]_{q_{i}} v_{i}\right) \\
& \sum_{r_{i}=0}^{\infty} r_{i}^{4} \frac{X_{r_{i}, q_{i}}\left(\left[m_{i}\right]_{q_{i}} v_{i}\right)}{\left[r_{i}\right]_{q_{i}} !} \\
&=\left[\left[m_{i}\right]_{q_{i}}^{4} X_{q_{i}}(1) v_{i}^{4}+4\left[m_{i}\right]_{q_{i}}^{3} X_{q_{i}}^{\prime}(1) v_{i}^{3}+6\left[m_{i}\right]_{q_{i}}^{2} X_{q_{i}}^{\prime \prime}(1) v_{i}^{2}+4\left[m_{i}\right]_{q_{i}} X_{q_{i}}^{\prime \prime \prime}(1) v_{i}+X_{q_{i}}^{(4)}(1)\right] e_{q_{i}}\left(\left[m_{i}\right] q_{q_{i}} v_{i}\right) .
\end{aligned}
$$

Lemma 3. Let $i, j=\{0,1,2,3,4\}$ and $f(t, s)=\lambda_{i, j}$ be the test functions such that $\lambda_{i, j}=t^{i} s^{j}$. Then, for every $m_{1}, m_{2} \in \mathbb{N} \backslash\{1,2,3,4\}$, operators $J_{m_{1}}^{q_{1}, q_{2}} m_{2}(. ;$. .) have the following equalities:

$$
\begin{aligned}
& \text { (1) } J_{m_{1}, m_{2}}^{q_{1}, q_{2}}\left(\lambda_{0,0} ; v_{1}, v_{2}\right)=\mathcal{C}_{m_{1}, r_{1}}^{q_{1}}\left(\lambda_{0,0} ; v_{1}, v_{2}\right) \\
& =\mathcal{D}_{m_{2}, r_{2}}^{q_{2}}\left(\lambda_{0,0} ; v_{1}, v_{2}\right)=1 \\
& \text { (2) } J_{m_{1}, m_{2}}^{q_{1}, q_{2}}\left(\lambda_{1,0} ; v_{1}, v_{2}\right)=\mathcal{C}_{m_{1}, r_{1}}^{q_{1}}\left(\lambda_{1,0} ; v_{1}, v_{2}\right) \\
& =\frac{1}{q_{1}\left[m_{1}-1\right]_{q_{1}}}+\frac{1}{\left[m_{1}-1\right]_{q_{1}}}\left(\left[m_{1}\right]_{q_{1}} v_{1}+\frac{X_{q_{1}}^{\prime}(1)}{X_{q_{1}}(1)}\right) ; \\
& \text { (3) } J_{m_{1}, m_{2}}^{q_{1}, q_{2}}\left(\lambda_{0,1} ; v_{1}, v_{2}\right)=\mathcal{D}_{m_{2}, r_{2}}^{q_{2}}\left(\lambda_{0,1} ; v_{1}, v_{2}\right) \\
& =\frac{1}{q_{2}\left[m_{2}-1\right]_{q_{2}}}+\frac{1}{\left[m_{2}-1\right]_{q_{2}}}\left(\left[m_{2}\right]_{q_{2}} v_{2}+\frac{X_{q_{2}}^{\prime}(1)}{X_{q_{2}}(1)}\right) ; \\
& \text { (4) } J_{m_{1}, m_{2}}^{q_{1}, q_{2}}\left(\lambda_{2,0} ; v_{1}, v_{2}\right)=\mathcal{C}_{m_{1}, r_{1}}^{q_{1}}\left(\lambda_{2,0} ; v_{1}, v_{2}\right) \\
& =\frac{\left(1+q_{1}\right)}{q_{1}^{3}\left[m_{1}-1\right]_{q_{1}}\left[m_{1}-2\right]_{q_{1}}}+\frac{\left(1+2 q_{1}\right)}{q_{1}^{2}\left[m_{1}-1\right]_{q_{1}}\left[m_{1}-2\right]_{q_{1}}}\left(\left[m_{1}\right]_{q_{1}} v_{1}+\frac{X_{q_{1}}^{\prime}(1)}{X_{q_{1}}(1)}\right)
\end{aligned}
$$




$$
+\frac{1}{\left[m_{1}-1\right]_{q_{1}}\left[m_{1}-2\right]_{q_{1}}}\left(\left[m_{1}\right]_{q_{1}}^{2} v_{1}^{2}+\frac{2\left[m_{1}\right]_{q_{1}} X_{q_{1}}^{\prime}(1)}{X_{q_{1}}(1)} v_{1}+\frac{X_{q_{1}}^{\prime \prime}(1)}{X_{q_{1}}(1)}\right) ;
$$

(5) $J_{m_{1}, m_{2}}^{q_{1}, q_{2}}\left(\lambda_{0,2} ; v_{1}, v_{2}\right)=\mathcal{D}_{m_{2}, r_{2}}^{q_{2}}\left(\lambda_{0,2} ; v_{1}, v_{2}\right)$

$$
\begin{aligned}
& =\frac{\left(1+q_{2}\right)}{q_{2}^{3}\left[m_{2}-1\right]_{q_{2}}\left[m_{2}-2\right]_{q_{2}}}+\frac{\left(1+2 q_{2}\right)}{q_{2}^{2}\left[m_{2}-1\right]_{q_{2}}\left[m_{2}-2\right]_{q_{2}}}\left(\left[m_{2}\right]_{q_{2}} v_{2}+\frac{X_{q_{2}}^{\prime}(1)}{X_{q_{2}}(1)}\right) \\
& +\frac{1}{\left[m_{2}-1\right]_{q_{2}}\left[m_{2}-2\right]_{q_{2}}}\left(\left[m_{2}\right]_{q_{2}}^{2} v_{2}^{2}+\frac{2\left[m_{2}\right]_{q_{2}} X_{q_{2}}^{\prime}(1)}{X_{q_{2}}(1)} v_{2}+\frac{X_{q_{2}}^{\prime \prime}(1)}{X_{q_{2}}(1)}\right) ;
\end{aligned}
$$

(6) $J_{m_{1}, m_{2}}^{q_{1}, q_{2}}\left(\lambda_{3,0} ; v_{1}, v_{2}\right)=\mathcal{C}_{m_{1}, r_{1}}^{q_{1}}\left(\lambda_{3,0} ; v_{1}, v_{2}\right)$

$$
\begin{aligned}
= & \frac{1+2 q_{1}+2 q_{1}^{2}+q_{1}^{3}}{q_{1}^{4}\left[m_{1}-1\right]_{q_{1}}\left[m_{1}-2\right]_{q_{1}}\left[m_{1}-3\right]_{q_{1}}} \\
& +\frac{\left(1+3 q_{1}+4 q_{1}^{2}+3 q_{1}^{3}\right)}{q_{1}^{3}\left[m_{1}-1\right]_{q_{1}}\left[m_{1}-2\right]_{q_{1}}\left[m_{1}-3\right]_{q_{1}}}\left(\left(\left[m_{1}\right]_{q_{1}} v_{1}+\frac{X_{q_{1}}^{\prime}(1)}{X_{q_{1}}(1)}\right)\right. \\
+ & \frac{\left(1+2 q_{1}+3 q_{1}^{2}\right)}{q_{1}\left[m_{1}-1\right]_{q_{1}}\left[m_{1}-2\right]_{q_{1}}\left[m_{1}-3\right]_{q_{1}}}\left(\left[m_{1}\right]_{q_{1}}^{2} v_{1}^{2}+\frac{2\left[m_{1}\right]_{q_{1}} X_{q_{1}}^{\prime}(1)}{X_{q_{1}}(1)} v_{1}\right. \\
& \left.+\frac{X_{q_{1}}^{\prime \prime}(1)}{X_{q_{1}}(1)}\right)+\frac{q_{1}^{2}}{\left[m_{1}-1\right]_{q_{1}}\left[m_{1}-2\right]_{q_{1}}\left[m_{1}-3\right]_{q_{1}}}\left(\left[m_{1}\right]_{q_{1}}^{3} v_{1}^{3}\right. \\
& \left.+3\left[m_{1}\right]_{q_{1}}^{2} \frac{X_{q_{1}}^{\prime}(1)}{X_{q_{1}}(1)} v_{1}^{2}+3\left[m_{1}\right]_{q_{1}} \frac{X_{q_{1}}^{\prime \prime}(1)}{X_{q_{1}}(1)} v_{1}+\frac{X_{q_{1}}^{\prime \prime}(1)}{X_{q_{1}}(1)}\right)
\end{aligned}
$$

(7) $J_{m_{1}, m_{2}}^{q_{1}, q_{2}}\left(\lambda_{0,3} ; v_{1}, v_{2}\right)=\mathcal{D}_{m_{2}, r_{2}}^{q_{2}}\left(\lambda_{0,3} ; v_{1}, v_{2}\right)$

$$
\begin{aligned}
= & \frac{1+2 q_{2}+2 q_{2}^{2}+q_{2}^{3}}{q_{2}^{4}\left[m_{2}-1\right]_{q_{2}}\left[m_{2}-2\right]_{q_{2}}\left[m_{2}-3\right]_{q_{2}}} \\
& +\frac{\left(1+3 q_{2}+4 q_{2}^{2}+3 q_{2}^{3}\right)}{q_{2}^{3}\left[m_{2}-1\right]_{q_{2}}\left[m_{2}-2\right]_{q_{2}}\left[m_{2}-3\right]_{q_{2}}}\left(\left(\left[m_{2}\right]_{q_{2}} v_{2}+\frac{X_{q_{2}}^{\prime}(1)}{X_{q_{2}}(1)}\right)\right. \\
+ & \frac{\left(1+2 q_{2}+3 q_{2}^{2}\right)}{q_{2}\left[m_{2}-1\right]_{q_{2}}\left[m_{2}-2\right]_{q_{2}}\left[m_{2}-3\right]_{q_{2}}}\left(\left[m_{2}\right]_{q_{2}}^{2} v_{2}^{2}+\frac{2\left[m_{2}\right]_{q_{2}} X_{q_{2}}^{\prime}(1)}{X_{q_{2}}(1)} v_{2}\right. \\
& \left.+\frac{X_{q_{2}}^{\prime \prime}(1)}{X_{q_{2}}(1)}\right)+\frac{q_{2}^{2}}{\left[m_{2}-1\right]_{q_{2}}\left[m_{2}-2\right]_{q_{2}}\left[m_{2}-3\right]_{q_{2}}}\left(\left[m_{2}\right]_{q_{2}}^{3} v_{2}^{3}\right. \\
& \left.+3\left[m_{2}\right]_{q_{2}}^{2} \frac{X_{q_{2}}^{\prime}(1)}{X_{q_{2}}(1)} v_{2}^{2}+3\left[m_{2}\right]_{q_{2}} \frac{X_{q_{2}}^{\prime \prime}(1)}{X_{q_{2}}(1)} v_{2}+\frac{X_{q_{2}}^{\prime \prime}(1)}{X_{q_{2}}(1)}\right) ;
\end{aligned}
$$

(8) $J_{m_{1}, m_{2}}^{q_{1}, q_{2}}\left(\lambda_{4,0} ; v_{1}, v_{2}\right)=\mathcal{C}_{m_{1}, r_{1}}^{q_{1}}\left(\lambda_{4,0} ; v_{1}, v_{2}\right)$

$$
\begin{aligned}
= & \frac{1+3 q_{1}+5 q_{1}^{2}+6 q_{1}^{3}+5 q_{1}^{4}+3 q_{1}^{5}+q_{1}^{6}}{q_{1}^{5}\left[m_{1}-1\right]_{q_{1}}\left[m_{1}-2\right]_{q_{1}}\left[m_{1}-3\right]_{q_{1}}\left[m_{1}-4\right]_{q_{1}}} \\
+ & \frac{\left(1+5 q_{1}+10 q_{1}^{2}+13 q_{1}^{3}+12 q_{1}^{4}+7 q_{1}^{5}+2 q_{1}^{6}\right)}{q_{1}^{3}\left[m_{1}-1\right]_{q_{1}}\left[m_{1}-2\right]_{q_{1}}\left[m_{1}-3\right]_{q_{1}}\left[m_{1}-4\right]_{q_{1}}}\left(\left[m_{1}\right]_{q_{1}} v_{1}+\frac{X_{q_{1}}^{\prime}(1)}{X_{q_{1}}(1)}\right) \\
+ & \frac{\left(1+3 q_{1}+7 q_{1}^{2}+9 q_{1}^{3}+9 q_{1}^{4}+6 q_{1}^{5}\right)}{q_{1}\left[m_{1}-1\right]_{q_{1}}\left[m_{1}-2\right]_{q_{1}}\left[m_{1}-3\right]_{q_{1}}\left[m_{1}-4\right]_{q_{1}}}\left(\left[m_{1}\right]_{q_{1}}^{2} v_{1}^{2}\right. \\
& \left.+\frac{2\left[m_{1}\right]_{q_{1}} X_{q_{1}}^{\prime}(1)}{X_{q_{1}}(1)} v_{1}+\frac{X_{q_{1}}^{\prime \prime}(1)}{X_{q_{1}}(1)}\right) \\
+ & \frac{\left(q_{1}^{2}+2 q_{1}^{3}+2 q_{1}^{4}+2 q_{1}^{5}+q_{1}^{6}+2 q_{1}^{7}\right)}{\left[m_{1}-1\right]_{q_{1}}\left[m_{1}-2\right]_{q_{1}}\left[m_{1}-3\right]_{q_{1}}\left[m_{1}-4\right]_{q_{1}}}\left(\left[m_{1}\right]_{q_{1}}^{3} v_{1}^{3}\right. \\
& \left.+3\left[m_{1}\right]_{q_{1}}^{2} \frac{X_{q_{1}}^{\prime}(1)}{X_{q_{1}}(1)} v_{1}^{2}+3\left[m_{1}\right]_{q_{1}} \frac{X_{q_{1}}^{\prime \prime}(1)}{X_{q_{1}}(1)} v_{1}+\frac{X_{q_{1}}^{\prime \prime \prime}(1)}{X_{q_{1}}(1)}\right)
\end{aligned}
$$


Mathematics 2022, 10, 675

6 of 21

$$
\begin{aligned}
& +\frac{q_{1}^{6}}{\left[m_{1}-1\right]_{q_{1}}\left[m_{1}-2\right]_{q_{1}}\left[m_{1}-3\right]_{q_{1}}\left[m_{1}-4\right]_{q_{1}}}\left(\left[m_{1}\right]_{q_{1}}^{4} v_{1}^{4}+4\left[m_{1}\right]_{q_{1}}^{3} \frac{X_{q_{1}}^{\prime}(1)}{X_{q_{1}}(1)} v_{1}^{3}\right. \\
& \left.+6\left[m_{1}\right]_{q_{1}}^{2} \frac{X_{q_{1}}^{\prime \prime}(1)}{X_{q_{1}}(1)} v_{1}^{2}+4\left[m_{1}\right]_{q_{1}} \frac{X_{q_{1}}^{\prime \prime \prime}(1)}{X_{q_{1}}(1)} v_{1}+\frac{X_{q_{1}}^{(4)}(1)}{X_{q_{1}}(1)}\right) ; \\
& J_{m_{1}, m_{2}}^{q_{1}, q_{2}}\left(\lambda_{0,4} ; v_{1}, v_{2}\right)=\mathcal{D}_{m_{2}, r_{2}}^{q_{2}}\left(\lambda_{0,4} ; v_{1}, v_{2}\right) \\
& =\frac{1+3 q_{2}+5 q_{2}^{2}+6 q_{2}^{3}+5 q_{2}^{4}+3 q_{2}^{5}+q_{2}^{6}}{q_{2}^{5}\left[m_{2}-1\right]_{q_{2}}\left[m_{2}-2\right]_{q_{2}}\left[m_{2}-3\right]_{q_{2}}\left[m_{2}-4\right]_{q_{2}}} \\
& +\frac{\left(1+5 q_{2}+10 q_{2}^{2}+13 q_{2}^{3}+12 q_{2}^{4}+7 q_{2}^{5}+2 q_{2}^{6}\right)}{q_{2}^{3}\left[m_{2}-1\right]_{q_{2}}\left[m_{2}-2\right]_{q_{2}}\left[m_{2}-3\right]_{q_{2}}\left[m_{2}-4\right]_{q_{2}}}\left(\left[m_{2}\right]_{q_{2}} v_{2}+\frac{X_{q_{2}}^{\prime}(1)}{X_{q_{2}}(1)}\right) \\
& +\frac{\left(1+3 q_{2}+7 q_{2}^{2}+9 q_{2}^{3}+9 q_{2}^{4}+6 q_{2}^{5}\right)}{q_{2}\left[m_{2}-1\right]_{q_{2}}\left[m_{2}-2\right]_{q_{2}}\left[m_{2}-3\right]_{q_{2}}\left[m_{2}-4\right]_{q_{2}}}\left(\left[m_{2}\right]_{q_{2}}^{2} v_{2}^{2}\right. \\
& \left.+\frac{2\left[m_{2}\right]_{q_{2}} X_{q_{2}}^{\prime}(1)}{X_{q_{2}}(1)} v_{2}+\frac{X_{q_{2}}^{\prime \prime}(1)}{X_{q_{2}}(1)}\right) \\
& +\frac{\left(q_{2}^{2}+2 q_{2}^{3}+2 q_{2}^{4}+2 q_{2}^{5}+q_{2}^{6}+2 q_{2}^{7}\right)}{\left[m_{2}-1\right]_{q_{2}}\left[m_{2}-2\right]_{q_{2}}\left[m_{2}-3\right]_{q_{2}}\left[m_{2}-4\right]_{q_{2}}}\left(\left[m_{2}\right]_{q_{2}}^{3} v_{2}^{3}\right. \\
& \left.+3\left[m_{2}\right]_{q_{2}}^{2} \frac{X_{q_{2}}^{\prime}(1)}{X_{q_{2}}(1)} v_{2}^{2}+3\left[m_{2}\right]_{q_{2}} \frac{X_{q_{2}}^{\prime \prime}(1)}{X_{q_{2}}(1)} v_{2}+\frac{X_{q_{2}}^{\prime \prime \prime}(1)}{X_{q_{2}}(1)}\right) \\
& +\frac{q_{2}^{6}}{\left[m_{2}-1\right]_{q_{2}}\left[m_{2}-2\right]_{q_{2}}\left[m_{2}-3\right]_{q_{2}}\left[m_{2}-4\right]_{q_{2}}}\left(\left[m_{2}\right]_{q_{2}}^{4} v_{2}^{4}+4\left[m_{2}\right]_{q_{2}}^{3} \frac{X_{q_{2}}^{\prime}(1)}{X_{q_{2}}(1)} v_{2}^{3}\right. \\
& \left.+6\left[m_{2}\right]_{q_{2}}^{2} \frac{X_{q_{2}}^{\prime \prime}(1)}{X_{q_{2}}(1)} v_{2}^{2}+4\left[m_{2}\right]_{q_{2}} \frac{X_{q_{2}}^{\prime \prime \prime}(1)}{X_{q_{2}}(1)} v_{2}+\frac{X_{q_{2}}^{(4)}(1)}{X_{q_{2}}(1)}\right) \text {. }
\end{aligned}
$$

Proof. For $i, j=0$, we take:

$$
\begin{aligned}
J_{m_{1}, m_{2}}^{q_{1}, q_{2}}\left(\lambda_{0,0} ; v_{1}, v_{2}\right)= & \sum_{r_{1}, r_{2}=0}^{\infty} \mathcal{U}_{m_{1}, q_{1}}^{r_{1}}\left(v_{1}\right) \mathcal{V}_{m_{2}, q_{2}}^{r_{2}}\left(v_{2}\right) \int_{0}^{\infty / A_{1} \infty / A_{2}} \int_{0}^{\infty} \mathcal{T}_{m_{1}, m_{2}}^{q_{1}, q_{2}}(t, s) d_{q_{1}} t d_{q_{2}} s \\
= & \sum_{r_{1}, r_{2}=0}^{\infty} \mathcal{U}_{m_{1}, q_{1}}^{r_{1}}\left(v_{1}\right) \mathcal{V}_{m_{2}, q_{2}}^{r_{2}}\left(v_{2}\right) \int_{0}^{\infty / A_{1}} \frac{t^{r_{1}}}{(1+t)_{q_{1}}^{r_{1}+m_{1}+1}} d_{q_{1}} t \int_{0}^{\infty / A_{2}} \frac{s^{r_{2}}}{(1+s)_{q_{2}}^{r_{2}+m_{2}+1}} d_{q_{2}} s \\
= & \frac{e_{q_{1}}\left(-\left[m_{1}\right]_{q_{1}} v_{1}\right)}{X_{q_{1}}(1)} \sum_{r_{1}=0}^{\infty} \frac{X_{r_{1}, q_{1}}\left(\left[m_{1}\right]_{q_{1}} v_{1}\right)}{\left[r_{1}\right]_{q_{1}} !} \frac{B_{q_{1}}\left(r_{1}+1, m_{1}\right)}{B_{q_{1}}\left(r_{1}+1, m_{1}\right)} \\
& \times \frac{e_{q_{2}}\left(-\left[m_{2}\right]_{q_{2}} v_{2}\right)}{X_{q_{2}}(1)} \sum_{r_{2}=0}^{\infty} \frac{X_{r_{2}, q_{2}}\left(\left[m_{2}\right]_{q_{2}} v_{2}\right)}{\left[r_{2}\right]_{q_{2}} !} \frac{B_{q_{2}}\left(r_{2}+1, m_{2}\right)}{B_{q_{2}}\left(r_{2}+1, m_{2}\right)} \\
= & 1 .
\end{aligned}
$$

For $i=1, j=0$, we obtain:

$$
\begin{aligned}
J_{m_{1}, m_{2}}^{q_{1}, q_{2}}\left(\lambda_{1,0} ; v_{1}, v_{2}\right)= & \sum_{r_{1}, r_{2}=0}^{\infty} \mathcal{U}_{m_{1}, q_{1}}^{r_{1}}\left(v_{1}\right) \mathcal{V}_{m_{2}, q_{2}}^{r_{2}}\left(v_{2}\right) \int_{0}^{\infty / A_{1}} \frac{\left(q_{1} t\right)^{r_{1}}}{(1+t)_{q_{1}}^{r_{1}+m_{1}+1}} d_{q_{1}} t \int_{0}^{\infty / A_{2}} \frac{s^{r_{2}}}{(1+s)_{q_{2}}^{r_{2}+m_{2}+1}} d_{q_{2}} s \\
= & \frac{e_{q_{1}}\left(-\left[m_{1}\right]_{q_{1}} v_{1}\right)}{X_{q_{1}}(1)} \sum_{r_{1}=0}^{\infty} \frac{X_{r_{1}, q_{1}}\left(\left[m_{1}\right]_{q_{1}} v_{1}\right)}{\left[r_{1}\right]_{q_{1}} !} q_{1}^{r_{1}} \frac{\mathcal{K}_{1}\left(A_{1}, r_{1}+1\right)}{B_{q_{1}}\left(r_{1}+1, m_{1}\right)} \int_{0}^{\infty / A_{1}} \frac{t^{r_{1}+1}}{(1+t)_{q_{1}}^{r_{1}+m_{1}+1}} d_{q_{1}} t \\
& \times \frac{e_{q_{2}}\left(-\left[m_{2}\right]_{q_{2}} v_{2}\right)}{X_{q_{2}}(1)} \sum_{r_{2}=0}^{\infty} \frac{X_{r_{2}, q_{2}}\left(\left[m_{2}\right]_{q_{2}} v_{2}\right)}{\left[r_{2}\right]_{q_{2}} !} \frac{B_{q_{2}}\left(r_{2}+1, m_{2}\right)}{B_{q_{2}}\left(r_{2}+1, m_{2}\right)}
\end{aligned}
$$




$$
\begin{aligned}
& =\frac{e_{q_{1}}\left(-\left[m_{1}\right]_{q_{1}} v_{1}\right)}{X_{q_{1}}(1)} \sum_{r_{1}=0}^{\infty} \frac{X_{r_{1}, q_{1}}\left(\left[m_{1}\right]_{q_{1}} v_{1}\right)}{\left[r_{1}\right]_{q_{1}} !} q_{1}^{r_{1}} \frac{\mathcal{K}_{1}\left(A_{1}, r_{1}+1\right)}{B_{q_{1}}\left(r_{1}+1, m_{1}\right)} \frac{B_{q_{1}}\left(r_{1}+2, m_{1}-1\right)}{\mathcal{K}_{1}\left(A_{1}, r_{1}+2\right)} \\
& =\frac{1}{q_{1}\left[m_{1}-1\right]_{q_{1}}} \frac{e_{q_{1}}\left(-\left[m_{1}\right]_{q_{1}} v_{1}\right)}{X_{q_{1}}(1)} \sum_{r_{1}=0}^{\infty} \frac{X_{r_{1}, q_{1}}\left(\left[m_{1}\right]_{q_{1}} v_{1}\right)}{\left[r_{1}\right]_{q_{1}} !}\left[r_{1}+1\right]_{q_{1}} .
\end{aligned}
$$

By applying:

$$
\begin{aligned}
{\left[r_{1}+1\right]_{q_{1}}=1+q_{1}\left[r_{1}\right]_{q_{1}}, } \\
J_{m_{1}, m_{2}, q_{2}}\left(\lambda_{1,0} ; v_{1}, v_{2}\right)=\frac{1}{q_{1}\left[m_{1}-1\right]_{q_{1}}} \frac{e_{q_{1}}\left(-\left[m_{1}\right]_{q_{1}} v_{1}\right)}{X_{q_{1}}(1)} \sum_{r_{1}=0}^{\infty} \frac{X_{r_{1}, q_{1}}\left(\left[m_{1}\right]_{q_{1}} v_{1}\right)}{\left[r_{1}\right]_{q_{1}} !} \\
+\frac{1}{\left[m_{1}-1\right]_{q_{1}}} \frac{e_{q_{1}}\left(-\left[m_{1}\right]_{q_{1}} v_{1}\right)}{X_{q_{1}}(1)} \sum_{r_{1}=0}^{\infty} \frac{X_{r_{1}, q_{1}}\left(\left[m_{1}\right]_{q_{1}} v_{1}\right)}{\left[r_{1}\right]_{q_{1}} !}\left[r_{1}\right]_{q_{1}} \\
=\frac{1}{q_{1}\left[m_{1}-1\right]_{q_{1}}}+\frac{1}{\left[m_{1}-1\right]_{q_{1}}}\left(\left[m_{1}\right]_{q_{1}} v_{1}+\frac{X_{q_{1}}^{\prime}(1)}{X_{q_{1}}(1)}\right)
\end{aligned}
$$

For $i=2, j=0$, we have:

$$
\begin{aligned}
J_{m_{1}, m_{2}}^{q_{1}, q_{2}}\left(\lambda_{2,0} ; v_{1}, v_{2}\right)= & \frac{e_{q}\left(-[m]_{q} x\right)}{X_{q}(1)} \sum_{r_{1}=0}^{\infty} \frac{X_{r_{1}, q_{1}}\left([m-1]_{q_{1}} v_{1}\right)}{\left[r_{1}\right]_{q_{1}} !} q_{1}^{2 r_{1}} \frac{\mathcal{K}_{1}\left(A_{1}, r_{1}+1\right)}{B_{q_{1}}\left(r_{1}+1, m_{1}\right)} \int_{0}^{\infty / A_{1}} \frac{t^{r_{1}+2}}{(1+t)_{q_{1}}^{r_{1}+m_{1}+1}} d_{q_{1}} t \\
& \times \frac{e_{q_{2}}\left(-\left[m_{2}\right]_{q_{2}} v_{2}\right)}{X_{q_{2}}(1)} \sum_{r_{2}=0}^{\infty} \frac{X_{r_{2}, q_{2}}\left(\left[m_{2}\right]_{q_{2}} v_{2}\right)}{\left[r_{2}\right]_{q_{2}} !} \frac{B_{q_{2}}\left(r_{2}+1, m_{2}\right)}{B_{q_{2}}\left(r_{2}+1, m_{2}\right)} \\
= & \frac{e_{q_{1}}\left(-\left[m_{1}\right]_{q_{1}} v_{1}\right)}{X_{q_{1}}(1)} \sum_{r_{1}=0}^{\infty} \frac{X_{r_{1}, q_{1}}\left(\left[m_{1}\right]_{q_{1}} v_{1}\right)}{\left[r_{1}\right]_{q_{1}} !} q_{1}^{2 r_{1}} \frac{\mathcal{K}_{1}\left(A_{1}, r_{1}+1\right)}{\mathcal{K}_{1}\left(A_{1}, r_{1}+3\right)} \frac{B_{q_{1}}\left(r_{1}+3, m_{1}-2\right)}{B_{q_{1}}\left(r_{1}+1, m_{1}\right)} \\
= & \frac{1}{q_{1}^{3}\left[m_{1}-1\right]_{q_{1}}\left[m_{1}-2\right]_{q_{1}}} \frac{e_{q_{1}}\left(-\left[m_{1}\right]_{q_{1}} v_{1}\right)}{X_{q_{1}}(1)} \sum_{r_{1}=0}^{\infty} \frac{X_{r_{1}, q_{1}}\left(\left[m_{1}\right]_{q_{1}} v_{1}\right)}{\left[r_{1}\right]_{q_{1}} !}\left[r_{1}+2\right]_{q_{1}}\left[r_{1}+1\right]_{q_{1}} .
\end{aligned}
$$

We use the equality (23) and $\left[r_{1}+2\right]_{q_{1}}=1+q_{1}+q_{1}^{2}\left[r_{1}\right]_{q_{1}}$ :

$$
\begin{aligned}
J_{m_{1}, m_{2}}^{q_{1}, q_{2}}\left(\lambda_{2,0} ; v_{1}, v_{2}\right)= & \frac{1}{q_{1}^{3}\left[m_{1}-1\right]_{q_{1}}\left[m_{1}-2\right]_{q_{1}}} \frac{e_{q_{1}}\left(-\left[m_{1}\right]_{q_{1}} v_{1}\right)}{X_{q_{1}}(1)} \sum_{r_{1}=0}^{\infty} \frac{X_{r_{1}, q_{1}}\left(\left[m_{1}\right]_{q_{1}} v_{1}\right)}{\left[r_{1}\right]_{q_{1}} !} \\
= & \frac{\left.\left(1+q_{1}\right)+q_{1}\left(1+2 q_{1}\right)\left[r_{1}\right]_{q_{1}}+q_{1}^{3}\left[r_{1}\right]_{q_{1}}^{2}\right)}{q_{1}^{3}\left[m_{1}-1\right]_{q_{1}}\left[m_{1}-2\right]_{q_{1}}}+\frac{\left(1+2 q_{1}\right)}{q_{1}^{2}\left[m_{1}-1\right]_{q_{1}}\left[m_{1}-2\right]_{q_{1}}}\left(\left(\left[m_{1}\right]_{q_{1}} v_{1}+\frac{X_{q_{1}}^{\prime}(1)}{X_{q_{1}}(1)}\right)\right. \\
& +\frac{1}{\left[m_{1}-1\right]_{q_{1}}\left[m_{1}-2\right]_{q_{1}}}\left(\left[m_{1}\right]_{q_{1}}^{2} v_{1}^{2}+\frac{2\left[m_{1}\right]_{q_{1}} X_{q_{1}}^{\prime}(1)}{X_{q_{1}}(1)} v_{1}+\frac{X_{q_{1}}^{\prime \prime}(1)}{X_{q_{1}}(1)}\right) .
\end{aligned}
$$

Similarly, we obtain the other identities. This completes the proof.

Lemma 4. Take $\Phi_{v_{1}, v_{2}}^{c, d}(t, s)=\left(t-v_{1}\right)^{c}\left(s-v_{2}\right)^{d}$ for all $c, d=\{0,1,2,3,4\}$. Then, for any $i=1,2, R_{r_{i}, q_{i}}\left(\left[m_{i}\right]_{q_{i}} v_{i}\right) \geq 0$ and $X_{q_{i}}(1) \neq 0$, suppose $\left(\delta_{m_{1}, m_{2}}^{c, d}\right)^{2}=J_{m_{1}, m_{2}}^{q_{1}, q_{2}}\left(\Phi_{v_{1}, v_{2}}^{c, d}(t, s) ; v_{1}, v_{2}\right)$.

Then, for all $i=1,2$, with $m_{i} \in \mathbb{N} \backslash\{1,2\}$, we obtain: 


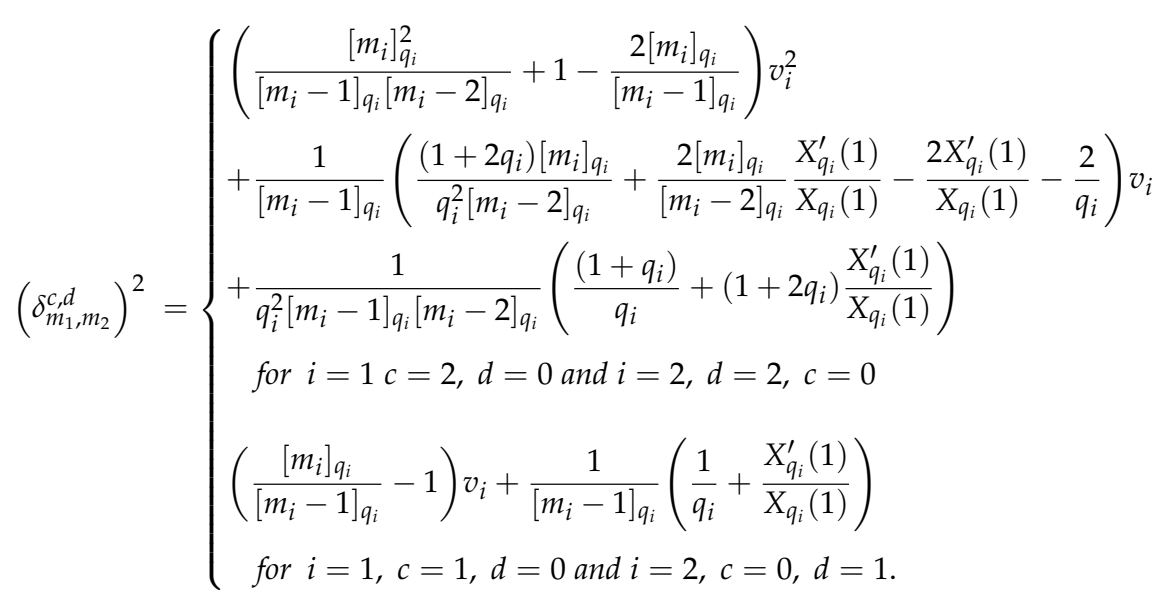

Remark 1. Let $m_{1}, m_{2} \in \mathbb{N}$, then, for any $\delta_{m_{1}}, \delta_{m_{2}}>0$, we take:

$$
\delta_{m_{1}, m_{2}}^{c, d}=\left\{\begin{array}{l}
\sqrt{\int_{m_{1}, m_{2}}^{q_{1}, q_{2}}\left(\Phi_{v_{1}, v_{2}}^{c, d}(t, s) ; v_{1}, v_{2}\right)}=\delta_{m_{1}} \text { (suppose) } \\
\text { for } c=2 \text { and } d=0 . \\
\sqrt{J_{m_{1}, m_{2}}^{q_{1}, q_{2}}\left(\Phi_{v_{1}, v_{2}}^{c, d}(t, s) ; v_{1}, v_{2}\right)}=\delta_{m_{2}} \text { (suppose) } \\
\text { for } d=2 \text { and } c=0 .
\end{array}\right.
$$

Lemma 5. For any $v_{1}, v_{2} \in \mathcal{M}^{2}$ and sufficiently large $m_{1}, m_{2} \in \mathbb{N} \backslash\{1,2,3,4\}$, the operators $J_{m_{1}, m_{2}}^{q_{1}, q_{2}}(. ;$.$) verify the following inequalities:$

(1) $J_{m_{1}, m_{2}}^{q_{1}, q_{2}}\left(\Phi_{v_{1}, v_{2}}^{2,0}(t, s) ; v_{1}, v_{2}\right)=O\left(\frac{1}{\left[m_{1}\right]_{q_{1}}^{2}}\right)\left(v_{1}+1\right)^{2} \leq M_{1}\left(v_{1}+1\right)^{2}$ as $m_{1}, m_{2} \rightarrow \infty$;

(2) $J_{m_{1}, m_{2}}^{q_{1}, q_{2}}\left(\Phi_{v_{1}, v_{2}}^{0,2}(t, s) ; v_{1}, v_{2}\right)=O\left(\frac{1}{\left[m_{2}\right]_{q_{2}}^{2}}\right)\left(v_{2}+1\right)^{2} \leq M_{2}\left(v_{2}+1\right)^{2}$ as $m_{1}, m_{2} \rightarrow \infty$;

(3) $J_{m_{1}, m_{2}}^{q_{1}, q_{2}}\left(\Phi_{v_{1}, v_{2}}^{4,0}(t, s) ; v_{1}, v_{2}\right)=O\left(\frac{1}{\left[m_{1}\right]_{q_{1}}^{4}}\right)\left(v_{1}+1\right)^{4} \leq M_{3}\left(v_{1}+1\right)^{4}$ as $m_{1}, m_{2} \rightarrow \infty$;

(4) $J_{m_{1}, m_{2}}^{q_{1}, q_{2}}\left(\Phi_{v_{1}, v_{2}}^{0,4}(t, s) ; v_{1}, v_{2}\right)=O\left(\frac{1}{\left[m_{2}\right]_{q_{2}}^{4}}\right)\left(v_{2}+1\right)^{4} \leq M_{4}\left(v_{2}+1\right)^{2}$ as $m_{1}, m_{2} \rightarrow \infty$.

\section{Approximation in Weighted Space and Degree of Convergence}

For any weight function $\psi$ given, such that $\psi\left(v_{1}, v_{2}\right)=1+v_{1}^{2}+v_{2}^{2}$, which satisfies the properties $B_{\psi}\left(\mathcal{M}^{2}\right)=\left\{f:\left|f\left(v_{1}, v_{2}\right)\right| \leq M_{f} \psi\left(v_{1}, v_{2}\right), \quad M_{f}>0\right\}$, where $B_{\psi}\left(\mathcal{M}^{2}\right)$ is defined for the class of all bounded functions on $\mathcal{M}^{2}=[0, \infty) \times[0, \infty)$. In addition, take $C^{(m)}\left(\mathcal{M}^{2}\right)$ as the $m$-times continuously differentiable functions on $\mathcal{M}^{2}=\left\{\left(v_{1}, v_{2}\right) \in \mathcal{M}^{2}:\right.$ $\left.v_{1}, v_{2} \in[0, \infty)\right\}$. The norm on $B_{\psi}$ is equipped by $\|f\|_{\psi}=\sup _{v_{1}, v_{2} \in \mathcal{M}^{2}} \frac{\left|f\left(v_{1}, v_{2}\right)\right|}{\psi\left(v_{1}, v_{2}\right)}$. Moreover, some classified functions are defined as follows:

$$
\begin{gathered}
C_{\psi}^{m}\left(\mathcal{M}^{2}\right)=\left\{f: f \in C_{\psi}\left(\mathcal{M}^{2}\right) ; \quad \text { such that } \lim _{\left(v_{1}, v_{2}\right) \rightarrow \infty} \frac{f\left(v_{1}, v_{2}\right)}{\psi\left(v_{1}, v_{2}\right)}=k_{f}<\infty\right\}, \\
C_{\psi}^{0}\left(\mathcal{M}^{2}\right)=\left\{f: f \in C_{\psi}^{m}\left(\mathcal{M}^{2}\right) ; \text { such that } \lim _{\left(v_{1}, v_{2}\right) \rightarrow \infty} \frac{f\left(v_{1}, v_{2}\right)}{\psi\left(v_{1}, v_{2}\right)}=0\right\},
\end{gathered}
$$




$$
C_{\psi}\left(\mathcal{M}^{2}\right)=\left\{f: f \in B_{\psi} \cap C_{\psi}\left(\mathcal{M}^{2}\right)\right\} .
$$

For all $f \in C_{\psi}^{0}\left(\mathcal{M}^{2}\right)$ and $\delta_{m_{1}}, \delta_{m_{2}}>0$, the weighted modulus of continuity of function $f$, suppose $\omega_{\psi}\left(f ; \delta_{m_{1}}, \delta_{m_{2}}\right)$ be defined by:

$$
\omega_{\psi}\left(f ; \delta_{m_{1}}, \delta_{m_{2}}\right)=\sup _{\left(v_{1}, v_{2}\right) \in[0, \infty)} \sup _{0 \leq\left|\varphi_{1}\right| \leq \delta_{m_{1}}, 0 \leq\left|\varphi_{2}\right| \leq \delta_{m_{2}}} \frac{\left|f\left(v_{1}+\varphi_{1}, v_{2}+\varphi_{2}\right)-f\left(v_{1}, v_{2}\right)\right|}{\psi\left(v_{1}, v_{2}\right) \psi\left(\varphi_{1}, \varphi_{2}\right)},
$$

for any $\mu_{1}, \mu_{2}>0$, one has:

$$
\begin{aligned}
& \omega_{\psi}\left(f ; \mu_{1} \delta_{m_{1}}, \mu_{2} \delta_{m_{2}}\right) \leq 4\left(1+\mu_{1}\right)\left(1+\mu_{2}\right)\left(1+\delta_{m_{1}}^{2}\right)\left(1+\delta_{m_{2}}^{2}\right) \omega_{\psi}\left(f ; \delta_{m_{1}}, \delta_{m_{2}}\right) \\
& \begin{aligned}
\left|f(t, s)-f\left(v_{1}, v_{2}\right)\right| & \leq \psi\left(v_{1}, v_{2}\right) \psi\left(\left|t-v_{1}\right|,\left|s-v_{2}\right|\right) \omega_{\psi}\left(f ;\left|t-v_{1}\right|,\left|s-v_{2}\right|\right) \\
& \leq\left(1+v_{1}^{2}+v_{2}^{2}\right)\left(1+\left(t-v_{1}\right)^{2}\right)\left(1+\left(s-v_{2}\right)^{2}\right) \omega_{\psi}\left(f ;\left|t-v_{1}\right|,\left|s-v_{2}\right|\right) .
\end{aligned}
\end{aligned}
$$

Theorem 1. Let $f \in C_{\psi}^{0}\left(\mathcal{M}^{2}\right)$. Then, for sufficiently large $m_{1}, m_{2} \in \mathbb{N}$, the operators $J_{m_{1}, m_{2}}^{q_{1}, q_{2}}$ satisfy the inequality:

$$
\begin{aligned}
\frac{\left|J_{m_{1}, m_{2}}^{q_{1}, q_{2}}\left(f ; v_{1}, v_{2}\right)-f\left(v_{1}, v_{2}\right)\right|}{4\left(1+v_{1}^{2}+v_{2}^{2}\right)} \leq & \Psi_{v_{1}, v_{2}}\left(1+O\left(\left[m_{1}\right]_{q_{1}}^{-2}\right)\right)\left(1+O\left(\left[m_{2}\right]_{q_{2}}^{-2}\right)\right) \\
& \times \omega_{\psi}\left(f ; O\left(\left[m_{1}\right]_{q_{1}}^{-1}\right), O\left(\left[m_{2}\right]_{q_{2}}^{-1}\right)\right),
\end{aligned}
$$

where $\Psi_{v_{1}, v_{2}}=\left(1+\left(v_{1}+1\right)+M_{1}\left(v_{1}+1\right)^{2}+M_{1}\left(v_{1}+1\right)^{3}\right)\left(1+\left(v_{2}+1\right)+M_{2}\left(v_{2}+1\right)^{2}+\right.$ $\left.M_{2}\left(v_{2}+1\right)^{3}\right) M_{1}, M_{2}>0$ and $\delta_{m_{i}}=O\left(\frac{1}{\left[m_{i}\right] q_{i}}\right)$, for $i=1,2$.

Proof. For all $\delta_{m_{1}}, \delta_{m_{2}}>0$, we have:

$$
\begin{aligned}
\left|f(t, s)-f\left(v_{1}, v_{2}\right)\right| & \leq 4\left(1+v_{1}^{2}+v_{2}^{2}\right)\left(1+\left(t-v_{1}\right)^{2}\right)\left(1+\left(s-v_{2}\right)^{2}\right) \\
& \times\left(1+\frac{\left|t-v_{1}\right|}{\delta_{m_{1}}}\right)\left(1+\frac{\left|s-v_{2}\right|}{\delta_{m_{2}}}\right)\left(1+\delta_{m_{1}}^{2}\right)\left(1+\delta_{m_{2}}^{2}\right) \omega_{\psi}\left(f ; \delta_{m_{1}}, \delta_{m_{2}}\right) \\
& =4\left(1+v_{1}^{2}+v_{2}^{2}\right)\left(1+\delta_{m_{1}}^{2}\right)\left(1+\delta_{m_{2}}^{2}\right) \\
& \times\left(1+\frac{\left|t-v_{1}\right|}{\delta_{m_{1}}}+\left(t-v_{1}\right)^{2}+\frac{1}{\delta_{m_{1}}}\left|t-v_{1}\right|\left(t-v_{1}\right)^{2}\right) \\
& \times\left(1+\frac{\left|s-v_{2}\right|}{\delta_{m_{2}}}+\left(s-v_{2}\right)^{2}+\frac{\left|s-v_{2}\right|}{\delta_{m_{2}}}\left(s-v_{2}\right)^{2}\right) \omega_{\psi}\left(f ; \delta_{m_{1}}, \delta_{m_{2}}\right) .
\end{aligned}
$$

On applying the positive linear operator $J_{m_{1}, m_{2}}^{q_{1}, q_{2}}$ and taking into account the well-known Cauchy-Schwarz inequality:

$$
\begin{aligned}
\frac{\left|J_{m_{1}, m_{2}}^{q_{1}, q_{2}}\left(f ; v_{1}, v_{2}\right)-f\left(v_{1}, v_{2}\right)\right|}{4\left(1+v_{1}^{2}+v_{2}^{2}\right)} \leq & J_{m_{1}, m_{2}}^{q_{1}, q_{2}}\left(\left(1+\frac{\left|t-v_{1}\right|}{\delta_{m_{1}}}+\left(t-v_{1}\right)^{2}\right.\right. \\
& \left.+\frac{1}{\delta_{m_{1}}}\left|t-v_{1}\right|\left(t-v_{1}\right)^{2} ; v_{1}, v_{2}\right) \\
\times & J_{m_{1}, m_{2}}^{q_{1}, q_{2}}\left(1+\frac{\left|s-v_{2}\right|}{\delta_{m_{2}}}+\left(s-v_{2}\right)^{2}+\frac{\left|s-v_{2}\right|}{\delta_{m_{2}}}\left(s-v_{2}\right)^{2} ; v_{1}, v_{2}\right) \\
\times & \left(1+\delta_{m_{1}}^{2}\right)\left(1+\delta_{m_{2}}^{2}\right) \omega_{\psi}\left(f ; \delta_{m_{1}}, \delta_{m_{2}}\right) \\
= & \left(1+v_{1}^{2}+v_{2}^{2}\right)\left(1+\delta_{m_{1}}^{2}\right)\left(1+\delta_{m_{2}}^{2}\right) \omega_{\psi}\left(f ; \delta_{m_{1}}, \delta_{m_{2}}\right)
\end{aligned}
$$




$$
\begin{aligned}
\times & \left(1+\frac{1}{\delta_{m_{1}}} J_{m_{1}, m_{2}}^{q_{1}, q_{2}}\left(\left|t-v_{1}\right| ; v_{1}, v_{2}\right)+J_{m_{1}, m_{2}}^{q_{1}, q_{2}}\left(\left(t-v_{1}\right)^{2} ; v_{1}, v_{2}\right)\right. \\
& +\frac{1}{\delta_{m_{1}}} J_{m_{1}, m_{2}}^{q_{1}, q_{2}}\left(\left|t-v_{1}\right|\left(t-v_{1}\right)^{2} ; v_{1}, v_{2}\right) \\
\times & \left(1+\frac{1}{\delta_{m_{2}}} J_{m_{1}, m_{2}}^{q_{1}, q_{2}}\left(\left|s-v_{2}\right| ; v_{1}, v_{2}\right)+J_{m_{1}, m_{2}}^{q_{1}, q_{2}}\left(\left(s-v_{2}\right)^{2} ; v_{1}, v_{2}\right)\right. \\
& +\frac{1}{\delta_{m_{2}}} J_{m_{1}, m_{2}}^{q_{1}, q_{2}}\left(\left|s-v_{2}\right|\left(s-v_{2}\right)^{2} ; v_{1}, v_{2}\right) ; \\
\leq & \left(1+\delta_{m_{1}}^{2}\right)\left(1+\delta_{m_{2}}^{2}\right) \omega_{\psi}\left(f ; \delta_{m_{1}}, \delta_{m_{2}}\right) \\
\left|J_{m_{1}, m_{2}}^{q_{1}, q_{2}}\left(f ; v_{1}, v_{2}\right)-f\left(v_{1}, v_{2}\right)\right| & \times\left[1+\frac{1}{\delta_{m_{1}}} \sqrt{J_{m_{1}, m_{2}}^{q_{1}, q_{2}}\left(\left(t-v_{1}\right)^{2} ; v_{1}, v_{2}\right)}+J_{m_{1}, m_{2}}^{q_{1}, q_{2}}\left(\left(t-v_{1}\right)^{2} ; v_{1}, v_{2}\right)\right. \\
4\left(1+v_{1}^{2}+v_{2}^{2}\right) & \left.+\frac{1}{\delta_{m_{1}}} \sqrt{J_{m_{1}, m_{2}}^{q_{1}, q_{2}}\left(\left(t-v_{1}\right)^{2} ; v_{1}, v_{2}\right)} \sqrt{J_{m_{1}, m_{2}}^{q_{1}, q_{2}}\left(\left(t-v_{1}\right)^{4} ; v_{1}, v_{2}\right)}\right] \\
\times & {\left[1+\frac{1}{\delta_{m_{2}}} \sqrt{J_{m_{1}, m_{2}}^{q_{1}, q_{2}}\left(\left(s-v_{2}\right)^{2} ; v_{1}, v_{2}\right)}+J_{m_{1}, m_{2}}^{q_{1}, q_{2}}\left(\left(s-v_{2}\right)^{2} ; v_{1}, v_{2}\right)\right.} \\
+ & \left.\frac{1}{\delta_{m_{2}}} \sqrt{J_{m_{1}, m_{2}}^{q_{1}, q_{2}}\left(\left(s-v_{2}\right)^{2} ; v_{1}, v_{2}\right)} \sqrt{J_{m_{1}, m_{2}}^{q_{1}, q_{2}}\left(\left(s-v_{2}\right)^{4} ; v_{1}, v_{2}\right)}\right] .
\end{aligned}
$$

Take into account Lemma 5 and for all $i=1,2$, if we choose $\delta_{m_{i}}=O\left(\frac{1}{\left.\left[m_{i}\right]\right]_{i}}\right)$, then:

$$
\begin{aligned}
\frac{\left|J_{m_{1}, m_{2}}^{q_{1}, q_{2}}\left(f ; v_{1}, v_{2}\right)-f\left(v_{1}, v_{2}\right)\right|}{4\left(1+v_{1}^{2}+v_{2}^{2}\right)} \leq & \left(1+\delta_{m_{1}}^{2}\right)\left(1+\delta_{m_{2}}^{2}\right) \omega_{\psi}\left(f ; \delta_{m_{1}}, \delta_{m_{2}}\right) \\
\times & {\left[1+\frac{1}{\delta_{m_{1}}} O\left(\frac{1}{\left[m_{1}\right]_{q_{1}}}\right)\left(v_{1}+1\right)+O\left(\frac{1}{\left[m_{1}\right]_{q_{1}}^{2}}\right)\left(v_{1}+1\right)^{2}\right.} \\
& \left.+\frac{1}{\delta_{m_{1}}} O\left(\frac{1}{\left[m_{1}\right]_{q_{1}}}\right)\left(v_{1}+1\right) O\left(\frac{1}{\left[m_{1}\right]_{q_{1}}^{2}}\right)\left(v_{1}+1\right)^{2}\right] \\
\times & {\left[1+\frac{1}{\delta_{m_{2}}} O\left(\frac{1}{\left[m_{2}\right]_{q_{2}}}\right)\left(v_{2}+1\right)+O\left(\frac{1}{\left[m_{2}\right]_{q_{2}}^{2}}\right)\left(v_{2}+1\right)^{2}\right.} \\
& \left.+\frac{1}{\delta_{m_{2}}} O\left(\frac{1}{\left[m_{2}\right]_{q_{2}}}\right)\left(v_{2}+1\right) O\left(\frac{1}{\left[m_{2}\right]_{q_{2}}^{2}}\right)\left(v_{2}+1\right)^{2}\right] \\
\leq & 4\left(1+O\left(\frac{1}{\left[m_{1}\right]_{q_{1}}^{2}}\right)\right)\left(1+O\left(\frac{1}{\left[m_{2}\right]_{q_{2}}^{2}}\right)\right) \\
\times & \omega_{\psi}\left(f ; O\left(\frac{1}{\left[m_{1}\right]_{q_{1}}}\right), O\left(\frac{1}{\left[m_{2}\right]_{q_{2}}}\right)\right) \\
\times & {\left[1+\left(v_{1}+1\right)+M_{1}\left(v_{1}+1\right)^{2}+M_{1}\left(v_{1}+1\right)^{3}\right] } \\
\times & {\left[1+\left(v_{2}+1\right)+M_{2}\left(v_{2}+1\right)^{2}+M_{2}\left(v_{2}+1\right)^{3}\right] . } \\
& {[1] }
\end{aligned}
$$

This completes the proof.

Lemma 6 ([31,32]). For the positive sequence of operators $\left\{L_{m_{1}, m_{2}}\right\}_{m_{1}, m_{2} \geq 1}$, which act as $C_{\psi} \rightarrow$ $B_{\psi}$, then there exists some positive $K$ such that:

$$
\left\|X_{m_{1}, m_{2}}\left(\psi ; v_{1}, v_{2}\right)\right\|_{\psi} \leq K .
$$


Theorem 2 ([31,32]). For the positive sequence of operators $\left\{X_{m_{1}, m_{2}}\right\}_{m_{1}, m_{2} \geq 1}$ acting as $C_{\psi} \rightarrow B_{\psi}$ defined earlier, the following conditions are satisfied:

$$
\begin{aligned}
& \text { (1) } \lim _{m_{1}, m_{2} \rightarrow \infty}\left\|X_{m_{1}, m_{2}}\left(1 ; v_{1}, v_{2}\right)-1\right\|_{\psi}=0 ; \\
& \text { (2) } \lim _{m_{1}, m_{2} \rightarrow \infty}\left\|X_{m_{1}, m_{2}}\left(t ; v_{1}, v_{2}\right)-v_{1}\right\|_{\psi}=0 ; \\
& \text { (3) } \lim _{m_{1}, m_{2} \rightarrow \infty}\left\|X_{m_{1}, m_{2}}\left(s ; v_{1}, v_{2}\right)-v_{2}\right\|_{\psi}=0 ;
\end{aligned}
$$$$
\text { (4) } \lim _{m_{1}, m_{2} \rightarrow \infty}\left\|X_{m_{1}, m_{2}}\left(\left(t^{2}+s^{2}\right) ; v_{1}, v_{2}\right)-\left(v_{1}^{2}+v_{2}^{2}\right)\right\|_{\psi}=0 \text {. }
$$

Then, for all $f \in C_{\psi}^{0}$ :

$$
\lim _{m_{1}, m_{2} \rightarrow \infty}\left\|X_{m_{1}, m_{2}} f-f\right\|_{\psi}=0
$$

and there exists another function $f \in C_{\psi} \backslash C_{\psi}^{0}$, such that:

$$
\lim _{m_{1}, m_{2} \rightarrow \infty}\left\|X_{m_{1}, m_{2}} f-f\right\|_{\psi} \geq 1 \text {. }
$$

Theorem 3. If $f \in C_{\psi}^{0}\left(\mathcal{M}^{2}\right)$, then we have:

$$
\lim _{m_{1}, m_{2} \rightarrow \infty}\left\|J_{m_{1}, m_{2}}^{q_{1}, q_{2}}(f)-f\right\|_{\psi}=0
$$

\section{Proof.}

$$
\begin{aligned}
& \left\|J_{m_{1}, m_{2}}^{q_{1}, q_{2}}\left(\psi ; v_{1}, v_{2}\right)\right\|_{\psi}=\sup _{\left(v_{1}, v_{2}\right) \in \mathcal{M}^{2}} \frac{\left|J_{m_{1}, m_{2}}^{q_{1}, q_{2}}\left(1+v_{1}^{2}+v_{2}^{2} ; v_{1}, v_{2}\right)\right|}{1+v_{1}^{2}+v_{2}^{2}} \\
& =1+\sup _{\left(v_{1}, v_{2}\right) \in \mathcal{M}^{2}}\left[\frac{1}{1+v_{1}^{2}+v_{2}^{2}}\left|\left(J_{m_{1}, m_{2}}^{q_{1}, q_{2}}\left(v_{1}^{2} ; v_{1}, v_{2}\right)+J_{m_{1}, m_{2}}^{q_{1}, q_{2}}\left(v_{2}^{2} ; v_{1}, v_{2}\right)\right)\right|\right] \\
& =1+\left|\frac{\left[m_{1}\right]_{q_{1}}^{2}}{\left[m_{1}-1\right]_{q_{1}}\left[m_{2}-1\right]_{q_{1}}}\right| \sup _{\left(v_{1}, v_{2}\right) \in \mathcal{M}^{2}} \frac{v_{1}^{2}}{1+v_{1}^{2}+v_{2}^{2}} \\
& +\left|\frac{\left(1+2 q_{1}\right)+2 q_{1}^{2} \frac{X_{q_{1}}^{\prime}(1)}{X_{q_{1}}(1)}}{q_{1}^{2}\left[m_{1}-1\right]_{q_{1}}\left[m_{2}-1\right]_{q_{1}}}\right| \sup _{\left(v_{1}, v_{2}\right) \in \mathcal{M}^{2}} \frac{v_{1}}{1+v_{1}^{2}+v_{2}^{2}} \\
& +\left|\frac{\left(1+2 q_{1}\right)+q_{1}\left(1+2 q_{1}\right) \frac{X_{q_{1}}^{\prime}(1)}{X_{q_{1}}(1)}+q_{1}^{3} \frac{X_{q_{1}}^{\prime \prime}(1)}{X_{q_{1}}(1)}}{q_{1}^{3}\left[m_{1}-1\right]_{q_{1}}\left[m_{2}-1\right]_{q_{1}}}\right| \sup _{\left(v_{1}, v_{2}\right) \in \mathcal{M}^{2}} \frac{1}{1+v_{1}^{2}+v_{2}^{2}} \\
& +\left|\frac{\left[m_{2}\right]_{q_{2}}^{2}}{\left[m_{1}-1\right]_{q_{2}}\left[m_{2}-1\right]_{q_{2}}}\right| \sup _{\left(v_{1}, v_{2}\right) \in \mathcal{M}^{2}} \frac{v_{2}^{2}}{1+v_{1}^{2}+v_{2}^{2}} \\
& +\left|\frac{\left(1+2 q_{2}\right)+2 q_{2}^{2} \frac{X_{q_{2}}^{\prime}(1)}{X_{q_{2}}(1)}}{q_{2}^{2}\left[m_{1}-1\right]_{q_{2}}\left[m_{2}-1\right]_{q_{2}}}\right| \sup _{\left(v_{1}, v_{2}\right) \in \mathcal{M}^{2}} \frac{v_{2}}{1+v_{1}^{2}+v_{2}^{2}} \\
& +\left|\frac{\left(1+2 q_{2}\right)+q_{2}\left(1+2 q_{2}\right) \frac{X_{q_{2}}^{\prime}(1)}{X_{q_{2}}(1)}+q_{2}^{3} \frac{X_{q_{2}}^{\prime \prime}(1)}{X_{q_{2}}(1)}}{q_{2}^{3}\left[m_{1}-1\right]_{q_{2}}\left[m_{2}-1\right]_{q_{2}}}\right| \sup _{\left(v_{1}, v_{2}\right) \in \mathcal{M}^{2}} \frac{1}{1+v_{1}^{2}+v_{2}^{2}} .
\end{aligned}
$$

Now, for all $m_{1}, m_{2} \in \mathbb{N} \backslash\{1,2\}$, there exists a positive constant $K$ such that:

$$
\left\|J_{m_{1}, m_{2}}^{q_{1}, q_{2}}\left(\psi ; v_{1}, v_{2}\right)\right\|_{\psi} \leq K
$$

Therefore, in order to prove Theorem 3, it is sufficient to take from the Lemma 3 and Theorem 2. Thus, we aimed to prove of Theorem 3. 
Here, we obtain the approximations of our new operators by the use of the secondorder modulus of continuity. For this purpose, if any $f \in C\left(\mathcal{M}^{2}\right)$ and $\delta>0$, then the second-order modulus of continuity in terms of partial continuity is given by:

$$
\omega\left(f ; \delta_{m_{1}}, \delta_{m_{2}}\right)=\sup \left\{\left|f(t, s)-f\left(v_{1}, v_{2}\right)\right|:(t, s),\left(v_{1}, v_{2}\right) \in \mathcal{M}^{2}\right\}
$$

with $\left|t-v_{1}\right| \leq \delta_{m_{1}},\left|s-v_{2}\right| \leq \delta_{m_{2}}$ with the partial modulus of continuity defined as:

$$
\begin{aligned}
& \omega_{1}\left(f ; \delta_{m_{1}}\right)=\sup _{0 \leq v_{1}, v_{2} \leq 1} \sup _{\left|x_{1}-x_{2}\right| \leq \delta_{m_{1}}}\left\{\left|f\left(x_{1}, v_{2}\right)-f\left(x_{2}, v_{2}\right)\right|\right\}, \\
& \omega_{2}\left(f ; \delta_{m_{2}}\right)=\sup _{0 \leq v_{1}, v_{2} \leq 1} \sup _{\left|y_{1}-y_{2}\right| \leq \delta_{m_{2}}}\left\{\left|f\left(v_{1}, y_{1}\right)-f\left(v_{1}, y_{2}\right)\right|\right\} .
\end{aligned}
$$

While for any function $\varphi$ and $\delta^{*}>0$, one has taken the modulus of continuity of order one $\omega^{*}\left(f ; \delta^{*}\right)$, and it is defined such that $\lim _{\delta^{*} \rightarrow 0+} \omega^{*}\left(\varphi ; \delta^{*}\right)=0$ and:

$$
\begin{gathered}
\omega^{*}\left(\varphi ; \delta^{*}\right)=\sup _{\left|v_{1}-v_{2}\right| \leq \delta^{*}}\left|\varphi\left(v_{1}\right)-\varphi\left(v_{2}\right)\right| ; \quad v_{1}, v_{2} \in[0, \infty), \\
\left|\varphi\left(v_{1}\right)-\varphi\left(v_{2}\right)\right| \leq\left(1+\frac{\left|v_{1}-v_{2}\right|}{\delta^{*}}\right) \omega^{*}\left(\varphi ; \delta^{*}\right) .
\end{gathered}
$$

Theorem 4. If $f \in C\left(\mathcal{M}^{2}\right)$, then we obtain the inequality for the operators $J_{m_{1}, m_{2}}^{q_{1}, q_{2}}$ :

$$
\left|J_{m_{1}, m_{2}}^{q_{1}, q_{2}}\left(f ; v_{1}, v_{2}\right)-f\left(v_{1}, v_{2}\right)\right| \leq 2\left(\omega_{1}\left(f ; \delta_{m_{1}, m_{2}}^{2,0}\right)+\omega_{2}\left(f ; \delta_{m_{1}, m_{2}}^{0,2}\right)\right) .
$$

Proof. For proof of Theorem 4, we use the Cauchy-Schwarz inequality, therefore, we obtain here:

$$
\begin{aligned}
\left|J_{m_{1}, m_{2}}^{q_{1}, q_{2}}\left(f ; v_{1}, v_{2}\right)-f\left(v_{1}, v_{2}\right)\right| & \leq J_{m_{1}, q_{2}}^{q_{1}, q_{2}}\left(\left|f(t, s)-f\left(v_{1}, v_{2}\right)\right| ; v_{1}, v_{2}\right) \\
& \leq J_{m_{1}, q_{2}}^{q_{1}, m_{2}}\left(\left|f(t, s)-f\left(v_{1}, s\right)\right| ; v_{1}, v_{2}\right) \\
& +J_{m_{1}}^{q_{1}, q_{2}}\left(v_{2}\left(\left|f\left(v_{1}, s\right)-f\left(v_{1}, v_{2}\right)\right| ; v_{1}, v_{2}\right)\right. \\
& \leq J_{m_{1}, m_{2}}^{q_{1}, q_{2}}\left(\omega_{1}\left(f ;\left|t-v_{1}\right|\right) ; v_{1}, v_{2}\right)+J_{m_{1}, m_{2}}^{q_{1}, q_{2}}\left(\omega_{2}\left(f ;\left|s-v_{2}\right|\right) ; v_{1}, v_{2}\right) \\
& \leq \omega_{1}\left(f ; \delta_{m_{1}}\right)\left(1+\delta_{m_{1}}^{-1} J_{m_{1}, q_{2}, q_{2}}\left(\left|t-v_{1}\right| ; v_{1}, v_{2}\right)\right) \\
& +\omega_{2}\left(f ; \delta_{m_{2}}\right)\left(1+\delta_{m_{2}}^{-1} J_{m_{1}, q_{2}, m_{2}}\left(\left|s-v_{2}\right| ; v_{1}, v_{2}\right)\right) \\
& \leq \omega_{1}\left(f ; \delta_{m_{1}}\right)\left(1+\frac{1}{\delta_{m_{1}}} \sqrt{J_{m_{1}, m_{2}}^{q_{1}, q_{2}}\left(\left(t-v_{1}\right)^{2} ; v_{1}, v_{2}\right)}\right) \\
& +\omega_{2}\left(f ; \delta_{m_{2}}\right)\left(1+\frac{1}{\delta_{m_{2}}} \sqrt{J_{m_{1}, m_{2}}^{q_{1}, q_{2}}\left(\left(s-v_{2}\right)^{2} ; v_{1}, v_{2}\right)}\right)
\end{aligned}
$$

where $\delta_{m_{1}}=\delta_{m_{1}, m_{2}}^{2,0}=\sqrt{J_{m_{1}, m_{2}}^{q_{1}, q_{2}}\left(\left(t-v_{1}\right)^{2} ; v_{1}, v_{2}\right)}$ and $\delta_{m_{2}}=$ $\delta_{m_{1}, m_{2}}^{0,2}=\sqrt{J_{m_{1}, m_{2}}^{q_{1}, q_{2}}\left(\left(s-v_{2}\right)^{2} ; v_{1}, v_{2}\right)}$, which then led to results.

Next, we obtain the convergence by use of class of the Lipschitz maximal in bivariate form for any function $f \in C\left(\mathcal{M}^{2}\right)$. For any positive constant $K$ and $0 \leq \rho_{1}, \rho_{2} \leq 1$ the Lipschitz maximal function on any set $\mathcal{I} \times \mathcal{I} \subset \mathcal{M}^{2}$ is given by:

$$
\begin{aligned}
\mathcal{L}_{\rho_{1}, \rho_{2}}(\mathcal{I} \times \mathcal{I}) & =\left\{f: \sup (1+t)^{\rho_{1}}(1+s)^{\rho_{2}}\left(f_{\rho_{1}, \rho_{2}}(t, s)-f_{\rho_{1}, \rho_{2}}\left(v_{1}, v_{2}\right)\right)\right. \\
& \left.\leq K \frac{1}{\left(1+v_{1}\right)^{\rho_{1}}} \frac{1}{\left(1+v_{2}\right)^{\rho_{2}}}\right\} .
\end{aligned}
$$




$$
f_{\rho_{1}, \rho_{2}}(t, s)-f_{\rho_{1}, \rho_{2}}\left(v_{1}, v_{2}\right)=\frac{\left|f(t, s)-f\left(v_{1}, v_{2}\right)\right|}{\left|t-v_{1}\right|^{\rho_{1} \mid}\left|s-v_{2}\right|^{\rho_{2}}} ; \quad(t, s),\left(v_{1}, v_{2}\right) \in \mathcal{M}^{2} .
$$

Theorem 5. For all $f \in \mathcal{L}_{\rho_{1}, \rho_{2}}(\mathcal{I} \times \mathcal{I})$, if $0 \leq \rho_{1}, \rho_{2} \leq 1$, then we obtain:

$$
\begin{aligned}
\left|J_{m_{1}, m_{2}}^{q_{1}, q_{2}}\left(f ; v_{1}, v_{2}\right)-f\left(v_{1}, v_{2}\right)\right| & \leq K\left\{\left(\left(d\left(v_{1}, \mathcal{I}\right)\right)^{\rho_{1}}+\left(\delta_{m_{1}, m_{2}}^{2,0}\right)^{\frac{\rho_{1}}{2}}\right)\left(\left(d\left(v_{2}, \mathcal{I}\right)\right)^{\rho_{2}}+\left(\delta_{m_{1}, m_{2}}^{0,2}\right)^{\frac{\rho_{2}}{2}}\right)\right. \\
& \left.+\left(d\left(v_{1}, \mathcal{I}\right)\right)^{\rho_{1}}\left(d\left(v_{2}, \mathcal{I}\right)\right)^{\rho_{2}}\right\},
\end{aligned}
$$

where $\delta_{m_{1}, m_{2}}^{2,0}$ and $\delta_{m_{1}, m_{2}}^{0,2}$ are defined by Theorem 4 and $K>0$.

Proof. Take into account $\left|v_{1}-x_{0}\right|=d\left(v_{1}, \mathcal{I}\right)$ and $\left|v_{2}-y_{0}\right|=d\left(v_{2}, E\right)$. For any $\left(v_{1}, v_{2}\right) \in$ $\mathcal{M}^{2}$ and $\left(x_{0}, y_{0}\right) \in \mathcal{I} \times \mathcal{I}$, we let $d\left(v_{1}, \mathcal{I}\right)=\inf \left\{\left|v_{1}-v_{2}\right|: v_{2} \in \mathcal{I}\right\}$. Thus, we can write here:

$$
\left|f(t, s)-f\left(v_{1}, v_{2}\right)\right| \leq K\left|f(t, s)-f\left(x_{0}, y_{0}\right)\right|+\left|f\left(x_{0}, y_{0}\right)-f\left(v_{1}, v_{2}\right)\right| .
$$

Applying $J_{m_{1}, m_{2}}^{q_{1}, q_{2}}$, we obtain:

$$
\begin{aligned}
\left|J_{m_{1}, m_{2}}^{q_{1}, q_{2}}\left(f ; v_{1}, v_{2}\right)-f\left(v_{1}, v_{2}\right)\right| & \leq J_{m_{1}, m_{2}}^{q_{1}, q_{2}}\left(\left|f\left(v_{1}, v_{2}\right)-f\left(x_{0}, y_{0}\right)\right|+\left|f\left(x_{0}, y_{0}\right)-f\left(v_{1}, v_{2}\right)\right|\right) \\
& \leq K J_{m_{1}, q_{2}}^{q_{1}}\left(\left|t-x_{0}\right|^{\rho_{1}}\left|s-y_{0}\right|^{\rho_{2}} ; v_{1}, v_{2}\right) \\
& +K\left|v_{1}-x_{0}\right|^{\rho_{1}}\left|v_{2}-y_{0}\right|^{\rho_{2}} .
\end{aligned}
$$

For all $U, V \geq 0$ and $\rho \in[0,1]$, we know the inequality $(U+V)^{\rho} \leq U^{\rho}+V^{\rho}$. Then, it is easy to obtain:

$$
\begin{aligned}
& \left|t-x_{0}\right|^{\rho_{1}} \leq\left|t-v_{1}\right|^{\rho_{1}}+\left|v_{1}-x_{0}\right|^{\rho_{1}}, \\
& \left|s-y_{0}\right|^{\rho_{2}} \leq\left|s-v_{2}\right|^{\rho_{2}}+\left|v_{2}-y_{0}\right|^{\rho_{2}} .
\end{aligned}
$$

Therefore:

$$
\begin{aligned}
\left|J_{m_{1}, m_{2}}^{q_{1}, q_{2}}\left(f ; v_{1}, v_{2}\right)-f\left(v_{1}, v_{2}\right)\right| & \leq K J_{m_{1}, m_{2}}^{q_{1}, q_{2}}\left(\left|t-v_{1}\right|^{\rho_{1}}\left|s-v_{2}\right|^{\rho_{2}} ; v_{1}, v_{2}\right) \\
& +K\left|v_{1}-x_{0}\right|^{\rho_{1}} J_{m_{1}}^{q_{1}, q_{2}}\left(\left|s-v_{2}\right|^{\rho_{2}} ; v_{1}, v_{2}\right) \\
& +K\left|v_{2}-y_{0}\right|^{\rho_{2}} J_{m_{1}, q_{2}}^{q_{1}, q_{2}}\left(\left|t-v_{1}\right|^{\rho_{1}} ; v_{1}, v_{2}\right) \\
& +2 K\left|v_{1}-x_{0}\right|^{\rho_{1}}\left|v_{2}-y_{0}\right|^{\rho_{2}} J_{m_{1}, m_{2}}^{q_{1}, q_{2}}\left(\lambda_{0,0} ; v_{1}, v_{2}\right),
\end{aligned}
$$

by the use of the Hölder inequality, we obtain the equality:

$$
\begin{aligned}
J_{m_{1}, m_{2}}^{q_{1}, q_{2}}\left(\left|t-v_{1}\right|^{\rho_{1}}\left|s-v_{2}\right|^{\rho_{2}} ; v_{1}, v_{2}\right) & =\mathcal{C}_{m_{1}, r_{1}}^{q_{1}}\left(\left|t-v_{1}\right|^{\rho_{1}} ; v_{1}, v_{2}\right) \mathcal{D}_{m_{2}, r_{2}}^{q_{2}}\left(\left|s-v_{2}\right|^{\rho_{2}} ; v_{1}, v_{2}\right) \\
& \leq\left(J_{m_{1}, m_{2}}^{q_{1}, q_{2}}\left(\left|t-v_{1}\right|^{2} ; v_{1}, v_{2}\right)\right)^{\frac{\rho_{1}}{2}}\left(J_{m_{1}, m_{2}}^{q_{1}, q_{2}}\left(\lambda_{0,0} ; v_{1}, v_{2}\right)\right)^{\frac{2-\rho_{1}}{2}} \\
& \times\left(J_{m_{1}, m_{2}}^{q_{1}, q_{2}}\left(\left|s-v_{2}\right|^{2} ; v_{1}, v_{2}\right)\right)^{\frac{\rho_{2}}{2}}\left(J_{m_{1}, m_{2}}^{q_{1}, q_{2}}\left(\lambda_{0,0} ; v_{1}, v_{2}\right)\right)^{\frac{2-\rho_{2}}{2}} .
\end{aligned}
$$

Thus, it is easy to obtain:

$$
\begin{aligned}
\left|J_{m_{1}, m_{2}}^{q_{1}, q_{2}}\left(f ; v_{1}, v_{2}\right)-f\left(v_{1}, v_{2}\right)\right| & \leq K\left(\delta_{m_{1}, m_{2}}^{2,0}\right)^{\frac{\rho_{1}}{2}}\left(\delta_{m_{1}, m_{2}}^{0,2}\right)^{\frac{\rho_{2}}{2}}+2 K\left(d\left(v_{1}, \mathcal{I}\right)\right)^{\rho_{1}}\left(d\left(v_{2}, \mathcal{I}\right)\right)^{\rho_{2}} \\
& +K\left(d\left(v_{1}, \mathcal{I}\right)\right)^{\rho_{1}}\left(\delta_{m_{1}, m_{2}}^{2,0}\right)^{\frac{\rho_{2}}{2}}+K\left(d\left(v_{2}, \mathcal{I}\right)\right)^{\rho_{2}}\left(\delta_{m_{1}, m_{2}}^{0,2}\right)^{\frac{\rho_{1}}{2}}
\end{aligned}
$$

This completes the proof. 
Theorem 6. Suppose $f \in C^{\prime}\left(\mathcal{M}^{2}\right)$. Then, for all $\left(v_{1}, v_{2}\right) \in \mathcal{M}^{2}$, the operators $J_{m_{1}, m_{2}}^{q_{1}, q_{2}}$ have the equality:

$$
\left|J_{m_{1}, m_{2}}^{q_{1}, q_{2}}\left(f ; v_{1}, v_{2}\right)-f\left(v_{1}, v_{2}\right)\right| \leq\left\|f_{v_{1}}\right\|_{C\left(\mathcal{M}^{2}\right)}\left(\delta_{m_{1}, m_{2}}^{2,0}\right)^{\frac{1}{2}}+\left\|f_{v_{2}}\right\|_{C\left(\mathcal{M}^{2}\right)}\left(\delta_{m_{1}, m_{2}}^{0,2}\right)^{\frac{1}{2}},
$$

where $\delta_{m_{1}, m_{2}}^{2,0}$ and $\delta_{m_{1}, m_{2}}^{0,2}$ are given by Theorem 4 .

Proof. For any $f \in C^{\prime}\left(\mathcal{M}^{2}\right)$ and fixed $\left(v_{1}, v_{2}\right) \in \mathcal{M}^{2}$, we take:

$$
f(t, s)-f\left(v_{1}, v_{2}\right)=\int_{v_{1}}^{t} f_{\varrho}(\varrho, s) \mathrm{d} \varrho+\int_{v_{2}}^{s} f_{\mu}\left(v_{1}, \mu\right) \mathrm{d} \mu .
$$

Apply operators $J_{m_{1}, m_{2}}^{q_{1}, q_{2}}$ on both sides, then:

$$
J_{m_{1}, m_{2}}^{q_{1}, q_{2}}\left(f(t, s) ; v_{1}, v_{2}\right)-f\left(v_{1}, v_{2}\right)=J_{m_{1}, m_{2}}^{q_{1}, q_{2}}\left(\int_{v_{1}}^{t} f_{\varrho}(\varrho, s) \mathrm{d} \varrho ; v_{1}, v_{2}\right)+J_{m_{1}, m_{2}}^{q_{1}, q_{2}}\left(\int_{v_{2}}^{s} f_{\mu}\left(v_{1}, \mu\right) \mathrm{d} \mu ; v_{1}, v_{2}\right) .
$$

The sup-norm on $\mathcal{M}^{2}$ gives us:

$$
\begin{gathered}
\left|\int_{v_{1}}^{t} f_{\varrho}(\varrho, s) \mathrm{d} \varrho\right| \leq \int_{v_{1}}^{t}\left|f_{\varrho}(\varrho, s) \mathrm{d} \varrho\right| \leq\left\|f_{v_{1}}\right\|_{C\left(\mathcal{M}^{2}\right)}\left|t-v_{1}\right| \\
\left|\int_{v_{2}}^{s} f_{\mu}\left(v_{1}, \mu\right) \mathrm{d} \mu\right| \leq \int_{v_{2}}^{s}\left|f_{\mu}\left(v_{1}, \mu\right) \mathrm{d} \mu\right| \leq\left\|f_{v_{2}}\right\| \|_{C\left(\mathcal{M}^{2}\right)}\left|s-v_{2}\right| .
\end{gathered}
$$

Taking into account (39)-(41), it is easy to obtain:

$$
\begin{aligned}
\left|J_{m_{1}, m_{2}}^{q_{1}, q_{2}}\left(f\left(v_{1}, v_{2}\right) ; v_{1}, v_{2}\right)-f\left(v_{1}, v_{2}\right)\right| & \leq J_{m_{1}, m_{2}}^{q_{1}, q_{2}}\left(\left|\int_{v_{1}}^{t} f_{\varrho}(\varrho, s) \mathrm{d} \varrho\right| ; v_{1}, v_{2}\right) \\
& +J_{m_{1}, m_{2}}^{q_{1}, q_{2}}\left(\left|\int_{v_{2}}^{s} f_{\mu}\left(v_{1}, \mu\right) \mathrm{d} \mu\right| ; v_{1}, v_{2}\right) \\
& \leq\left\|f_{v_{1}}\right\|_{C\left(\mathcal{M}^{2}\right)} J_{m_{1}, m_{2}}^{q_{1}, q_{2}}\left(\left|t-v_{1}\right| ; v_{1}, v_{2}\right) \\
& +\left\|f_{v_{2}}\right\|_{C\left(\mathcal{M}^{2}\right)} J_{m_{1}, m_{2}}^{q_{1}, q_{2}}\left(\left|s-v_{2}\right| ; v_{1}, v_{2}\right) \\
& \leq\left\|f_{v_{1}}\right\|_{C\left(\mathcal{M}^{2}\right)}\left(J_{m_{1}, m_{2}}^{q_{1}, q_{2}}\left(\left(t-v_{1}\right)^{2} ; v_{1}, v_{2}\right) J_{m_{1}, m_{2}}^{q_{1}, q_{2}}\left(1 ; v_{1}, v_{2}\right)\right)^{\frac{1}{2}} \\
& +\left\|f_{v_{2}}\right\|_{C\left(\mathcal{M}^{2}\right)}\left(J_{m_{1}, m_{2}}^{q_{1}, q_{2}}\left(\left(s-v_{2}\right)^{2} ; v_{1}, v_{2}\right) J_{m_{1}, m_{2}}^{q_{1}, q_{2}}\left(1 ; v_{1}, v_{2}\right)\right)^{\frac{1}{2}} \\
& =\left\|f_{v_{1}}\right\|_{C\left(\mathcal{M}^{2}\right)}\left(\delta_{m_{1}, m_{2}}^{2,0}\right){ }^{\frac{1}{2}}+\left\|f_{v_{2}}\right\|_{C\left(\mathcal{M}^{2}\right)}\left(\delta_{m_{1}, m_{2}}^{0,2}\right)^{\frac{1}{2}} .
\end{aligned}
$$

Theorem 7. For an arbitrary function $f \in C\left(\mathcal{M}^{2}\right)$, we define the auxiliary operators as:

$$
S_{m_{1}, m_{2}}^{q_{1}, q_{2}}\left(f ; v_{1}, v_{2}\right)=J_{m_{1}, m_{2}}^{q_{1}, q_{2}}\left(f ; v_{1}, v_{2}\right)+f\left(v_{1}, v_{2}\right)-f\left(\mathcal{C}_{m_{1}, r_{1}}^{q_{1}}\left(\lambda_{1,0} ; v_{1}, v_{2}\right), \mathcal{D}_{m_{2}, r_{2}}^{q_{2}}\left(\lambda_{1,0} ; v_{1}, v_{2}\right)\right)
$$

Then, for all $f \in C^{\prime}\left(\mathcal{M}^{2}\right)$, the operators $S_{m_{1}, m_{2}}^{q_{1}, q_{2}}$ verify the inequality:

$$
\begin{aligned}
S_{m_{1}, m_{2}}^{q_{1}, q_{2}}(f ; t, s)-f\left(v_{1}, v_{2}\right) & \leq\left\{\delta_{m_{1}, m_{2}}^{2,0}+\delta_{m_{1}, m_{2}}^{0,2}+\left(\mathcal{C}_{m_{1}, r_{1}}^{q_{1}}\left(\lambda_{1,0} ; v_{1}, v_{2}\right)-v_{1}\right)^{2}\right. \\
& \left.+\left(\mathcal{D}_{m_{2}, r_{2}}^{q_{2}}\left(\lambda_{1,0} ; v_{1}, v_{2}\right)-v_{2}\right)^{2}\right\}\|f\|_{C^{2}\left(\mathcal{M}^{2}\right)}
\end{aligned}
$$


where $\mathcal{C}_{m_{1}, r_{1}}^{q_{1}}\left(\lambda_{1,0} ; v_{1}, v_{2}\right)$ and $\mathcal{D}_{m_{2}, r_{2}}^{q_{2}}\left(\lambda_{0,1} ; v_{1}, v_{2}\right)$ are defined by Lemma 3.

Proof. By the use of Lemma 3, it is easy to obtain $S_{m_{1}, m_{2}}^{q_{1}, q_{2}}\left(1 ; v_{1}, v_{2}\right)=1, S_{m_{1}, m_{2}}^{q_{1}, q_{2}}\left(t-v_{1} ; v_{1}, v_{2}\right)=$ 0 and $S_{m_{1}, m_{2}}^{q_{1}, q_{2}}\left(s-v_{2} ; v_{1}, v_{2}\right)=0$. In addition, for any $f \in C^{\prime}\left(\mathcal{M}^{2}\right)$ from the Taylor series expansions, we obtain:

$$
\begin{aligned}
f(t, s)-f\left(v_{1}, v_{2}\right) & =\frac{\partial f\left(v_{1}, v_{2}\right)}{\partial v_{1}}\left(t-v_{1}\right)+\int_{v_{1}}^{t}(t-\chi) \frac{\partial^{2} f\left(\chi, v_{2}\right)}{\partial \chi^{2}} \mathrm{~d} \chi \\
& +\frac{\partial f\left(v_{1}, v_{2}\right)}{\partial v_{2}}\left(s-v_{2}\right)+\int_{v_{2}}^{s}(s-\sigma) \frac{\partial^{2} f\left(v_{1}, \sigma\right)}{\partial \sigma^{2}} \mathrm{~d} \sigma .
\end{aligned}
$$

On applying $S_{m_{1}, m_{2}}^{q_{1}, q_{2}}$, we see that:

$$
\begin{aligned}
S_{m_{1}, m_{2}}^{q_{1}, q_{2}}\left(f(t, s) ; v_{1}, v_{2}\right)-S_{m_{1}, m_{2}}^{q_{1}, q_{2}}\left(f\left(v_{1}, v_{2}\right)\right. \\
=S_{m_{1}, m_{2}}^{q_{1}, q_{2}}\left(\int_{v_{1}}^{t}(t-\chi) \frac{\partial^{2} f\left(\chi, v_{2}\right)}{\partial \chi^{2}} \mathrm{~d} \chi ; v_{1}, v_{2}\right)+S_{m_{1}, m_{2}}^{q_{1}, q_{2}}\left(\int_{v_{2}}^{s}(s-\sigma) \frac{\partial^{2} f\left(v_{1}, \sigma\right)}{\partial \sigma^{2}} \mathrm{~d} \sigma ; v_{1}, v_{2}\right) \\
=J_{m_{1}, m_{2}}^{q_{1}, q_{2}}\left(\int_{v_{1}}^{t}(t-\chi) \frac{\partial^{2} f\left(\chi, v_{2}\right)}{\partial \chi^{2}} \mathrm{~d} \chi ; v_{1}, v_{2}\right)+J_{m_{1}, m_{2}}^{q_{1}, q_{2}}\left(\int_{v_{2}}^{s}(s-\sigma) \frac{\partial^{2} f\left(v_{1}, \sigma\right)}{\partial \sigma^{2}} \mathrm{~d} \sigma ; v_{1}, v_{2}\right) \\
\quad-\int_{v_{1}}^{\mathcal{C}_{m_{1}, r_{1}}^{q_{1}}\left(\lambda_{1,0} ; v_{1}, v_{2}\right)}\left(\mathcal{C}_{m_{1}, r_{1}}^{q_{1}}\left(\lambda_{1,0} ; v_{1}, v_{2}\right)-\chi\right) \frac{\partial^{2} f\left(\chi, v_{2}\right)}{\partial \chi^{2}} \mathrm{~d} \chi \\
\quad-\int_{v_{2}}^{\mathcal{D}_{m_{2}, r_{2}}^{q_{2}}\left(\lambda_{1,0} ; v_{1}, v_{2}\right)}\left(\mathcal{D}_{m_{2}, r_{2}}^{q_{2}}\left(\lambda_{1,0} ; v_{1}, v_{2}\right)-\sigma\right) \frac{\partial^{2} f\left(v_{1}, \sigma\right)}{\partial \sigma^{2}} \mathrm{~d} \sigma .
\end{aligned}
$$

From this hypothesis, we easily obtain:

$$
\begin{aligned}
& \left|\int_{v_{1}}^{t}(t-\chi) \frac{\partial^{2} f\left(\chi, v_{2}\right)}{\partial \chi^{2}} \mathrm{~d} \chi\right| \leq \int_{v_{1}}^{t}\left|(t-\chi) \frac{\partial^{2} f\left(\chi, v_{2}\right)}{\partial \chi^{2}} \mathrm{~d} \chi\right| \leq\|f\|_{C^{2}\left(\mathcal{M}^{2}\right)}\left(t-v_{1}\right)^{2} \text {, } \\
& \left|\int_{v_{2}}^{s}(s-\sigma) \frac{\partial^{2} f\left(v_{1}, \sigma\right)}{\partial \sigma^{2}} \mathrm{~d} \sigma\right| \leq \int_{v_{2}}^{s}\left|(s-\sigma) \frac{\partial^{2} f\left(v_{1}, \sigma\right)}{\partial \sigma^{2}} \mathrm{~d} \sigma\right| \leq\|f\|_{C^{2}\left(\mathcal{M}^{2}\right)}\left(s-v_{2}\right)^{2} \text {, } \\
& \left|\int_{v_{1}}^{\mathcal{C}_{m_{1}, r_{1}}^{q_{1}}\left(\lambda_{1,0} ; v_{1}, v_{2}\right)}\left(\mathcal{C}_{m_{1}, r_{1}}^{q_{1}}\left(\lambda_{1,0} ; v_{1}, v_{2}\right)-\chi\right) \frac{\partial^{2} f\left(\chi, v_{2}\right)}{\partial \chi^{2}} \mathrm{~d} \chi\right| \\
& \leq\|f\|_{C^{2}\left(\mathcal{M}^{2}\right)}\left(\mathcal{C}_{m_{1}, r_{1}}^{q_{1}}\left(\lambda_{1,0} ; v_{1}, v_{2}\right)-v_{1}\right)^{2} \\
& \left|\int_{v_{2}}^{\mathcal{D}_{m_{2}, r_{2}}^{q_{2}}\left(\lambda_{1,0} ; v_{1}, v_{2}\right)}\left(\mathcal{D}_{m_{2}, r_{2}}^{q_{2}}\left(\lambda_{1,0} ; v_{1}, v_{2}\right)-\sigma\right) \frac{\partial^{2} f\left(v_{1}, \sigma\right)}{\partial \sigma^{2}} \mathrm{~d} \sigma\right| \\
& \leq\|f\|_{C^{2}\left(\mathcal{M}^{2}\right)}\left(\mathcal{D}_{m_{2}, r_{2}}^{q_{2}}\left(\lambda_{1,0} ; v_{1}, v_{2}\right)-v_{2}\right)^{2} \text {. }
\end{aligned}
$$

Thus:

$$
\left|S_{m_{1}, m_{2}}^{q_{1}, q_{2}}(f ; t, s)-f\left(v_{1}, v_{2}\right)\right| \leq\left\{J_{m_{1}, m_{2}}^{q_{1}, q_{2}}\left(\left(t-v_{1}\right)^{2} ; v_{1}, v_{2}\right)+J_{m_{1}, m_{2}}^{q_{1}, q_{2}}\left(\left(s-v_{2}\right)^{2} ; v_{1}, v_{2}\right)\right.
$$




$$
\begin{aligned}
& +\left(\mathcal{C}_{m_{1}, r_{1}}^{q_{1}}\left(\lambda_{1,0} ; v_{1}, v_{2}\right)-v_{1}\right)^{2} \\
& \left.+\left(\mathcal{D}_{m_{2}, r_{2}}^{q_{2}}\left(\lambda_{1,0} ; v_{1}, v_{2}\right)-v_{2}\right)^{2}\right\}\|f\|_{C^{2}\left(\mathcal{M}^{2}\right)},
\end{aligned}
$$

which completes the desired proof of Theorem 7.

\section{GBS-Type Approximation in Bögel Space}

Suppose $\mathcal{M}_{1} \times \mathcal{M}_{2}=\left\{\left(v_{1}, v_{2}\right): 0 \leq v_{1}, v_{2}<\infty\right\}$. Take any function $f: \mathcal{M}_{1} \times \mathcal{M}_{2} \rightarrow$ $\mathbb{R}$ for real compact intervals of $\mathcal{M}_{1} \times \mathcal{M}_{2}$. For all $(t, s),\left(v_{1}, v_{2}\right) \in \mathcal{M}_{1} \times \mathcal{M}_{2}$ suppose $\Delta_{(t, s)}^{*} f\left(v_{1}, v_{2}\right)$ denotes the bivariate mixed difference operators defined as follows:

$$
\Delta_{(t, s)}^{*} f\left(v_{1}, v_{2}\right)=f(t, s)-f\left(t, v_{2}\right)-f\left(v_{1}, s\right)+f\left(v_{1}, v_{2}\right) .
$$

If at any point $\left(v_{1}, v_{2}\right) \in \mathcal{M}_{1} \times \mathcal{M}_{2}$, the function $f: \mathcal{M}_{1} \times \mathcal{M}_{2} \rightarrow \mathbb{R}$ defined on $\mathcal{M}_{1} \times \mathcal{M}_{2}$, then $\lim _{(t, s) \rightarrow\left(v_{1}, v_{2}\right)} \Delta_{(t, s)}^{*} f\left(v_{1}, v_{2}\right)=0$.

If set of all the space of all Bögel-continuous (B-continuous) denoted by $C_{B}\left(\mathcal{M}_{1} \times \mathcal{M}_{2}\right)$ on $\left(v_{1}, v_{2}\right) \in \mathcal{M}_{1} \times \mathcal{M}_{2}$ and be defined such that $C_{B}\left(\mathcal{M}_{1} \times \mathcal{M}_{2}\right)=\{f$, such that $f$ : $\mathcal{M}_{1} \times \mathcal{M}_{2} \rightarrow \mathbb{R}$ is $f, B$ - bounded on $\left.\mathcal{M}_{1} \times \mathcal{M}_{2}\right\}$. Next, the Bögel-differentiable function on $\left(v_{1}, v_{2}\right) \in \mathcal{M}_{1} \times \mathcal{M}_{2}$ be $f: \mathcal{M}_{1} \times \mathcal{M}_{2} \rightarrow \mathbb{R}$ and limit exists finite defined by:

$$
\lim _{(t, s) \rightarrow\left(v_{1}, v_{2}\right), t \neq v_{1}, s \neq v_{2}} \frac{1}{\left(t-v_{1}\right)\left(s-v_{2}\right)}\left(\Delta_{(t, s)}^{*}\right)=D_{B} f\left(v_{1}, v_{2}\right)<\infty .
$$

Let the classes of all Bögel-differentiable function denoted by $D_{\psi} f\left(v_{1}, v_{2}\right)$ and be $D_{\psi}\left(\mathcal{M}_{1} \times \mathcal{M}_{2}\right)=\left\{f\right.$, such that $f: \mathcal{M}_{1} \times \mathcal{M}_{2} \rightarrow \mathbb{R}$ is $f$, B-differentiable on $\left.\mathcal{M}_{1} \times \mathcal{M}_{2}\right\}$. Suppose the function $f$ is $B$-bounded on $D$ and be $f: \mathcal{M}_{1} \times \mathcal{M}_{2} \rightarrow \mathbb{R}$, then for all $(t, s),\left(v_{1}, v_{2}\right) \in \mathcal{M}_{1} \times \mathcal{M}_{2}$ there exists positive constant $M$ such that $\left|\Delta_{(t, s)}^{*} f\left(v_{1}, v_{2}\right)\right| \leq M$. The classes of all $B$-continuous function is called a $B$-bounded on $\mathcal{M}_{1} \times \mathcal{M}_{2}$, when $\mathcal{M}_{1} \times$ $\mathcal{M}_{2}$ is compact subset. Let $B_{\psi}\left(\mathcal{M}_{1} \times \mathcal{M}_{2}\right)$ denote the classes of all $B$-bounded function defined on $\mathcal{M}_{1} \times \mathcal{M}_{2}$, which equipped the norm on $B$ as $\|f\|_{B}=\sup _{(t, s),\left(v_{1}, v_{2}\right) \in \mathcal{M}_{1} \times \mathcal{M}_{2}} \mid$ $\Delta_{(t, s)}^{*} f\left(v_{1}, v_{2}\right) \mid$. As we know to approximate the degree for a set of all $B$-continuous function on positive linear operators, it is essential to use the properties of mixed-modulus of continuity. So, we let for all $(t, s)$ and $\left(v_{1}, v_{2}\right) \in \mathcal{M}_{1} \times \mathcal{M}_{2}$ and $f \in B_{\psi}\left(\mathcal{M}_{1} \times \mathcal{M}_{2}\right)$, the mixed-modulus of continuity of function $f$ such that $\omega_{B}:[0, \infty) \times[0, \infty) \rightarrow \mathbb{R}^{2}$ and be defined such as:

$$
\omega_{B}\left(f ; \delta_{1}, \delta_{2}\right)=\sup \left\{\Delta_{(t, s)}^{*} f\left(v_{1}, v_{2}\right):\left|t-v_{1}\right| \leq \delta_{1},\left|s-v_{2}\right| \leq \delta_{2}\right\} .
$$

For any $\mathcal{M}_{1} \times \mathcal{M}_{2}=[0, \infty) \times[0, \infty)$, we suppose the classes of all $B$-continuous function defined on $\mathcal{M}_{1} \times \mathcal{M}_{2}$ denoted by $C_{\psi}\left(\mathcal{M}_{1} \times \mathcal{M}_{2}\right)$. Moreover, let set of all ordinary continuous function defined on $\mathcal{M}_{1} \times \mathcal{M}_{2}$ be $C\left(\mathcal{M}_{1} \times \mathcal{M}_{2}\right)$. For further details on space of Bögel functions and GBS-type operators related to this paper, we propose the article [33-38].

Let $\left(v_{1}, v_{2}\right) \in \mathcal{M}_{1} \times \mathcal{M}_{2}$ and $m_{1}, m_{2} \in \mathbb{N}$. Then, for all $f \in C\left(\mathcal{M}_{1} \times \mathcal{M}_{2}\right)$, we define the GBS-type new positive linear operators $K_{m_{1}, m_{2}}^{q_{1}, q_{2}}$ for the operators $J_{m_{1}, m_{2}}^{q_{1}, q_{2}}$. Thus, we suppose:

$$
K_{m_{1}, m_{2}}^{q_{1}, q_{2}}\left(f(t, s) ; v_{1}, v_{2}\right)=J_{m_{1}, m_{2}}^{q_{1}, q_{2}}\left(f\left(t, v_{2}\right)+f\left(v_{1}, s\right)-f(t, s) ; v_{1}, v_{2}\right) .
$$

More precisely, the generalized GBS operator for the bivariate function is defined as follows: 


$$
K_{m_{1}, m_{2}}^{q_{1}, q_{2}}\left(f(t, s) ; v_{1}, v_{2}\right)=\sum_{r_{1}, r_{2}=0}^{\infty} \mathcal{U}_{m_{1}, q_{1}}^{r_{1}}\left(v_{1}\right) \mathcal{V}_{m_{2}, q_{2}}^{r_{2}}\left(v_{2}\right) \int_{0}^{\infty / A_{1}} \int_{0}^{\infty / A_{2}} Q_{v_{1}, v_{2}}(t, s) f\left(q_{1}^{r_{1}} t, q_{2}^{r_{2}} s\right) d_{q_{1}} t d_{q_{2}} s,
$$

where $Q_{v_{1}, v_{2}}(t, s)=\mathcal{T}_{m_{1}, m_{2}}^{q_{1}, q_{2}}(t, s) P_{v_{1}, v_{2}}(t, s)$ and $P_{v_{1}, v_{2}}(t, s)=\left(f\left(t, v_{2}\right)+f\left(v_{1}, s\right)-f(t, s)\right)$.

Theorem 8. For all $f \in C_{\psi}\left(\mathcal{M}_{1} \times \mathcal{M}_{2}\right)$, the operators $K_{m_{1}, m_{2}}^{q_{1}, q_{2}}$ verify that:

$$
\left|K_{m_{1}, m_{2}}^{q_{1}, q_{2}}\left(f(t, s) ; v_{1}, v_{2}\right)-f\left(v_{1}, v_{2}\right)\right| \leq 4 \omega_{B}\left(f ; \delta_{m_{1}, m_{2}}^{2,0}, \delta_{m_{1}, m_{2}}^{0,2}\right)
$$

where $\delta_{m_{1}, m_{2}}^{2,0}$ and $\delta_{m_{1}, m_{2}}^{0,2}$ are defined by Theorem 4 .

Proof. Let $(t, s)$ and $\left(v_{1}, v_{2}\right) \in \mathcal{M}_{1} \times \mathcal{M}_{2}$. For all $m_{1}, m_{2} \in \mathbb{N}$ and $\delta_{m_{1}}, \delta_{m_{2}}>0$, it follows that:

$$
\begin{aligned}
\left|\Delta_{\left(v_{1}, v_{2}\right)}^{*} f(t, s)\right| & \leq \omega_{B}\left(f ;\left|t-v_{1}\right|\left|s-v_{2}\right|\right) \\
& \leq\left(1+\frac{t-v_{1}}{\delta_{m_{1}}}\right)\left(1+\frac{s-v_{2}}{\delta_{m_{2}}}\right) \omega_{B}\left(f ; \delta_{m_{1}}, \delta_{m_{2}}\right) .
\end{aligned}
$$

From (45) and the well-known Cauchy-Schwarz inequality, we easily conclude that:

$$
\begin{aligned}
\left|K_{m_{1}, m_{2}}^{q_{1}, q_{2}}\left(f(t, s) ; v_{1}, v_{2}\right)-f\left(v_{1}, v_{2}\right)\right| & \leq J_{m_{1}, m_{2}}^{q_{1}, q_{2}}\left(\left|\Delta_{\left(v_{1}, v_{2}\right)}^{*} f(t, s)\right| ; v_{1}, v_{2}\right) \\
& \leq\left(J_{m_{1}, m_{2}}^{q_{1}, q_{2}}\left(\lambda_{0,0} ; v_{1}, v_{2}\right)+\frac{1}{\delta_{m_{1}}}\left(J_{m_{1}, m_{2}}^{q_{1}, q_{2}}\left(\left(t-v_{1}\right)^{2} ; v_{1}, v_{2}\right)\right)^{\frac{1}{2}}\right. \\
& +\frac{1}{\delta_{m_{2}}}\left(J_{m_{1}, m_{2}}^{q_{1}, q_{2}}\left(\left(s-v_{2}\right)^{2} ; v_{1}, v_{2}\right)\right)^{\frac{1}{2}} \\
& +\frac{1}{\delta_{m_{1}}}\left(J_{m_{1}, m_{2}}^{q_{1}, q_{2}}\left(\left(t-v_{1}\right)^{2} ; v_{1}, v_{2}\right)\right)^{\frac{1}{2}} \\
& \left.\times \frac{1}{\delta_{m_{2}}}\left(J_{m_{1}, m_{2}}^{q_{1}, q_{2}}\left(\left(s-v_{2}\right)^{2} ; v_{1}, v_{2}\right)\right)^{\frac{1}{2}}\right) \omega_{B}\left(f ; \delta_{m_{1}}, \delta_{m_{2}}\right) .
\end{aligned}
$$

In the view of Theorem 4, we easily obtain our results.

If we let $x=(t, s), y=\left(v_{1}, v_{2}\right) \in \mathcal{M}_{1} \times \mathcal{M}_{2}$, then the Lipschitz function in terms of $B$-continuous functions is defined by:

$$
\operatorname{Lip}_{M}^{\xi}=\left\{f \in C\left(\mathcal{M}_{1} \times \mathcal{M}_{2}\right):\left|\Delta_{\left(v_{1}, v_{2}\right)}^{*} f(x, y)\right| \leq M\|x-y\|^{\xi}\right\}
$$

where $M$ is a positive constant, $0<\xi \leq 1$, and the Euclidean norm $\|x-y\|=$ $\sqrt{\left(t-v_{1}\right)^{2}+\left(s-v_{2}\right)^{2}}$.

Theorem 9. For all $f \in \operatorname{Lip} p_{M}^{\xi}$, the operators $K_{m_{1}, m_{2}}^{q_{1}, q_{2}}$ satisfy:

$$
\left|K_{m_{1}, m_{2}}^{q_{1}, q_{2}}\left(f(x, y) ; v_{1}, v_{2}\right)-f\left(v_{1}, v_{2}\right)\right| \leq M\left(\delta_{m_{1}, m_{2}}^{2,0}+\delta_{m_{1}, m_{2}}^{0,2}\right)^{\frac{\xi}{2}}
$$

where $\delta_{m_{1}, m_{2}}^{2,0}$ and $\delta_{m_{1}, m_{2}}^{0,2}$ are defined by Theorem 4 .

Proof. We easily see that:

$$
\begin{aligned}
K_{m_{1}, m_{2}}^{q_{1}, q_{2}}\left(f(x, y) ; v_{1}, v_{2}\right) & =J_{m_{1}, m_{2}}^{q_{1}, q_{2}}\left(f\left(v_{1}, y\right)+f\left(x, v_{2}\right)-f(x, s) ; v_{1}, v_{2}\right) \\
& =J_{m_{1}, m_{2}}^{q_{1}, q_{2}}\left(f\left(v_{1}, v_{2}\right)-\Delta_{\left(v_{1}, v_{2}\right)}^{*} f(x, s) ; v_{1}, v_{2}\right)
\end{aligned}
$$




$$
=f\left(v_{1}, v_{2}\right)-J_{m_{1}, m_{2}}^{q_{1}, q_{2}}\left(\Delta_{\left(v_{1}, v_{2}\right)}^{*} f(x, s) ; v_{1}, v_{2}\right)
$$

Therefore:

$$
\begin{aligned}
\left|K_{m_{1}, m_{2}}^{q_{1}, q_{2}}\left(f(x, y) ; v_{1}, v_{2}\right)-f\left(v_{1}, v_{2}\right)\right| & \leq J_{m_{1}, m_{2}}^{q_{1}, q_{2}}\left(\left|\Delta_{\left(v_{1}, v_{2}\right)}^{*} f(x, y)\right| ; v_{1}, v_{2}\right) \\
& \leq M J_{m_{1}, m_{2}}^{q_{1}, q_{2}}\left(\|x-y\|^{\xi} ; v_{1}, v_{2}\right) \\
& \leq M\left(J_{m_{1}, m_{2}}^{q_{1}, q_{2}}\left(\|x-y\|^{2} ; v_{1}, v_{2}\right)\right)^{\frac{\xi}{2}} \\
& \leq M\left(J_{m_{1}, m_{2}}^{q_{1}, q_{2}}\left(\left(t-v_{1}\right)^{2} ; v_{1}, v_{2}\right)+J_{m_{1}, m_{2}}^{q_{1}, q_{2}}\left(\left(s-v_{2}\right)^{2} ; v_{1}, v_{2}\right)\right)^{\frac{\xi}{2}} .
\end{aligned}
$$

Theorem 10. For all $f \in D_{\psi}\left(\mathcal{M}_{1} \times \mathcal{M}_{2}\right)$ and $D_{B} f \in B\left(\mathcal{M}_{1} \times \mathcal{M}_{2}\right)$, we obtain:

$$
\begin{aligned}
\left|K_{m_{1}, m_{2}}^{q_{1}, q_{2}}\left(f ; v_{1}, v_{2}\right)-f\left(v_{1}, v_{2}\right)\right| & \leq C\left\{1+3\left\|D_{B} f\right\|_{\infty}\right\}\left(v_{1}+1\right)\left(v_{2}+1\right) \\
& +\left\{1+\sqrt{M_{2}}\left(v_{1}+1\right)+\sqrt{M_{1}}\left(v_{2}+1\right)\right\}\left(v_{1}+1\right)\left(v_{2}+1\right) \\
& \times \omega_{\text {mixed }}\left(D_{B} f ; O\left(\frac{1}{\left[m_{1}\right]_{q_{1}}}\right), O\left(\frac{1}{\left[m_{2}\right]_{q_{2}}}\right)\right),
\end{aligned}
$$

where $C$ is any positive constant.

Proof. Suppose $\rho \in\left(v_{1}, t\right), \xi \in\left(v_{2}, s\right)$ and

$$
\begin{gathered}
\Delta_{\left(v_{1}, v_{2}\right)}^{*} f(t, s)=\left(t-v_{1}\right)\left(s-v_{2}\right) D_{B} f(\rho, \xi), \\
D_{B} f(\rho, \xi)=\Delta_{\left(v_{1}, v_{2}\right)}^{*} D_{B} f(\rho, \xi)+D_{B} f(\rho, y)+D_{B} f(x, \xi)-D_{B} f\left(v_{1}, v_{2}\right) .
\end{gathered}
$$

For all $D_{B} f \in B\left(\mathcal{M}_{1} \times \mathcal{M}_{2}\right)$, it follows that:

$$
\begin{aligned}
\left|K_{m_{1}, m_{2}}^{q_{1}, q_{2}}\left(\Delta_{\left(v_{1}, v_{2}\right)}^{*} f(t, s) ; v_{1}, v_{2}\right)\right| & =\left|K_{m_{1}, m_{2}}^{q_{1}, q_{2}}\left(\left(t-v_{1}\right)\left(s-v_{2}\right) D_{B} f(\rho, \xi) ; v_{1}, v_{2}\right)\right| \\
\leq & K_{m_{1}, m_{2}}^{q_{1}, q_{2}}\left(\left|t-v_{1}\right|\left|s-v_{2}\right|\left|\Delta_{\left(v_{1}, v_{2}\right)}^{*} D_{B} f(\rho, \xi)\right| ; v_{1}, v_{2}\right) \\
& +K_{m_{1}, m_{2}}^{q_{1}, q_{2}}\left(| t - v _ { 1 } | | s - v _ { 2 } | \left(\left|D_{B} f\left(\rho, v_{2}\right)\right|\right.\right. \\
& \left.\left.+\left|D_{B} f\left(v_{1}, \xi\right)\right|+\left|D_{B} f\left(v_{1}, v_{2}\right)\right|\right) ; v_{1}, v_{2}\right) \\
\leq & K_{m_{1}, m_{2}}^{q_{1}, q_{2}}\left(\left|t-v_{1}\right|\left|s-v_{2}\right|\right. \\
& \left.\times \omega_{\text {mixed }}\left(D_{B} f ;\left|\rho-v_{1}\right|,\left|\xi-v_{2}\right|\right) ; v_{1}, v_{2}\right) \\
& +3\left\|D_{B} f\right\| \|_{\infty} K_{m_{1}, m_{2}}^{q_{1}, q_{2}}\left(\left|t-v_{1} \| s-v_{2}\right| ; v_{1}, v_{2}\right) .
\end{aligned}
$$

Here, $\omega_{\text {mixed }}$ is the mixed-modulus of continuity, and it follows that:

$$
\begin{aligned}
\omega_{\text {mixed }}\left(D_{B} f ;\left|\rho-v_{1}\right|,\left|\xi-v_{2}\right|\right) \\
\quad \leq \omega_{\text {mixed }}\left(D_{B} f ;\left|t-v_{1}\right|,\left|s-v_{2}\right|\right) \\
\leq\left(1+\delta_{m_{1}}^{-1}\left|t-v_{1}\right|\right)\left(1+\delta_{m_{2}}^{-1}\left|s-v_{2}\right|\right) \omega_{\text {mixed }}\left(D_{B} f ; \mid \delta_{m_{1}}, \delta_{m_{2}}\right) .
\end{aligned}
$$


Therefore, it is obvious that:

$$
\begin{aligned}
& \left|K_{m_{1}, m_{2}}^{*}\left(f ; v_{1}, v_{2}\right)-f\left(v_{1}, v_{2}\right)\right|=\left|\Delta_{\left(v_{1}, v_{2}\right)}^{*} f(t, s) ; v_{1}, v_{2}\right| \\
& \leq 3\left\|D_{B} f\right\|_{\infty}\left(K_{m_{1}, m_{2}}^{q_{1}, q_{2}}\left(\left(t-v_{1}\right)^{2}\left(s-v_{2}\right)^{2} ; v_{1}, v_{2}\right)\right)^{\frac{1}{2}} \\
& +\left(K_{m_{1}, m_{2}}^{q_{1}, q_{2}}\left(\left|t-v_{1}\right|\left|s-v_{2}\right| ; v_{1}, v_{2}\right)\right. \\
& \left.+\delta_{m_{1}}^{-1} K_{m_{1}, m_{2}}^{q_{1}, q_{2}}\left(\left(t-v_{1}\right)^{2}\left|s-v_{2}\right| ; v_{1}, v_{2}\right)\right) \\
& +\delta_{m_{2}}^{-1} K_{m_{1}, m_{2}}^{q_{1}, q_{2}}\left(\left|t-v_{1}\right|\left(s-v_{2}\right)^{2} ; v_{1}, v_{2}\right) \\
& +\delta_{m_{1}}^{-1} \delta_{m_{2}}^{-1} K_{m_{1}, m_{2}}^{q_{1}, q_{2}}\left(\left(t-v_{1}\right)^{2}\left(s-v_{2}\right)^{2} ; v_{1}, v_{2}\right) \omega_{\text {mixed }}\left(D_{B} f ; \delta_{m_{1}}, \delta_{m_{2}}\right) \text {; } \\
& \left|K_{m_{1}, m_{2}}^{*}\left(f ; v_{1}, v_{2}\right)-f\left(v_{1}, v_{2}\right)\right|=\left|\Delta_{\left(v_{1}, v_{2}\right)}^{*} f(t, s) ; v_{1}, v_{2}\right| \\
& \leq 3\left\|D_{B} f\right\|_{\infty}\left(K_{m_{1}, m_{2}}^{q_{1}, q_{2}}\left(\Phi_{v_{1}, v_{2}}^{2,2} ; v_{1}, v_{2}\right)\right)^{\frac{1}{2}} \\
& +\left\{\left(K_{m_{1}, m_{2}}^{q_{1}, q_{2}}\left(\Phi_{v_{1}, v_{2}}^{2,2} ; v_{1}, v_{2}\right)\right)^{\frac{1}{2}}\right. \\
& +\delta_{m_{1}}^{-1}\left(K_{m_{1}, m_{2}}^{q_{1}, q_{2}}\left(\Phi_{v_{1}, v_{2}}^{4,2} ; v_{1}, v_{2}\right)\right)^{\frac{1}{2}}+\delta_{m_{2}}^{-1}\left(K_{m_{1}, m_{2}}^{q_{1}, q_{2}}\left(\Phi_{v_{1}, v_{2}}^{2,4} ; v_{1}, v_{2}\right)\right)^{\frac{1}{2}} \\
& \left.+\delta_{m_{1}}^{-1} \delta_{m_{2}}^{-1} K_{m_{1}, m_{2}}^{q_{1}, q_{2}}\left(\Phi_{v_{1}, v_{2}}^{2,2} ; v_{1}, v_{2}\right)\right\} \omega_{\text {mixed }}\left(D_{B} f ; \delta_{m_{1}}, \delta_{m_{2}}\right)
\end{aligned}
$$

which follows that:

$$
\begin{aligned}
\left|K_{m_{1}, m_{2}}^{*}\left(f ; v_{1}, v_{2}\right)-f\left(v_{1}, v_{2}\right)\right| & =3\left\|D_{B} f\right\|_{\infty}\left(K_{m_{1}, m_{2}}^{q_{1}, q_{2}}\left(\Phi_{v_{1}, v_{2}}^{2,0} ; v_{1}, v_{2}\right) K_{m_{1}, m_{2}}^{q_{1}, q_{2}}\left(\Phi_{v_{1}, v_{2}}^{0,2} ; v_{1}, v_{2}\right)\right)^{\frac{1}{2}} \\
& +\left\{\left(K_{m_{1}, m_{2}}^{q_{1}, q_{2}}\left(\Phi_{v_{1}, v_{2}}^{2,0} ; v_{1}, v_{2}\right) K_{m_{1}, m_{2}}^{q_{1}, q_{2}}\left(\Phi_{v_{1}, v_{2}}^{0,2} ; v_{1}, v_{2}\right)\right)^{\frac{1}{2}}\right. \\
& +\delta_{m_{1}}^{-1}\left(K_{m_{1}, m_{2}}^{q_{1}, q_{2}}\left(\Phi_{v_{1}, v_{2}}^{4,0} ; v_{1}, v_{2}\right) K_{m_{1}, m_{2}}^{q_{1}, q_{2}}\left(\Phi_{v_{1}, v_{2}}^{0,2} ; v_{1}, v_{2}\right)\right)^{\frac{1}{2}} \\
& +\delta_{m_{2}}^{-1}\left(K_{m_{1}, m_{2}}^{q_{1}, q_{2}}\left(\Phi_{v_{1}, v_{2}}^{2,0} ; v_{1}, v_{2}\right) K_{m_{1}, m_{2}}^{q_{1}, q_{2}}\left(\Phi_{v_{1}, v_{2}}^{0,4} ; v_{1}, v_{2}\right)\right)^{\frac{1}{2}} \\
& \left.+\delta_{m_{1}}^{-1} \delta_{m_{2}}^{-1} K_{m_{1}, m_{2}}^{q_{1}, q_{2}}\left(\Phi_{v_{1}, v_{2}}^{2,0} ; v_{1}, v_{2}\right) K_{m_{1}, m_{2}}^{q_{1}, q_{2}}\left(\Phi_{v_{1}, v_{2}}^{0,2} ; v_{1}, v_{2}\right)\right\} \\
& \times \omega_{\text {mixed }}\left(D_{B} f ; \delta_{m_{1}}, \delta_{m_{2}}\right) .
\end{aligned}
$$

From Lemma 5, we demonstrate:

$$
\begin{aligned}
\left|K_{m_{1}, m_{2}}^{*}\left(f ; v_{1}, v_{2}\right)-f\left(v_{1}, v_{2}\right)\right| \leq & 3\left\|D_{B} f\right\|_{\infty}\left(\sqrt{M_{1} M_{2}}\left(v_{1}+1\right)\left(v_{2}+1\right)\right) \\
+ & \left\{\left(\sqrt{M_{1} M_{2}}\left(v_{1}+1\right)\left(v_{2}+1\right)\right)\right. \\
+ & \delta_{m_{1}}^{-1}\left(\sqrt{M_{2}} O\left(\frac{1}{\left[m_{1}\right]_{q_{1}}}\right)\left(v_{1}+1\right)^{2}\left(v_{2}+1\right)\right) \\
+ & \delta_{m_{2}}^{-1}\left(\sqrt{M_{1}} O\left(\frac{1}{\left[m_{2}\right]_{q_{2}}}\right)\left(v_{2}+1\right)^{2}\left(v_{1}+1\right)\right) \\
+ & \left.\delta_{m_{1}}^{-1} \delta_{m_{2}}^{-1}\left(O\left(\frac{1}{\left[m_{1}\right]_{q_{1}}}\right) O\left(\frac{1}{\left[m_{2}\right]_{q_{2}}}\right)\left(v_{1}+1\right)\left(v_{2}+1\right)\right)\right\}
\end{aligned}
$$




$$
\times \omega_{\text {mixed }}\left(D_{B} f ; \delta_{m_{1}}, \delta_{m_{2}}\right),
$$

where, in the view of Theorem 1, we have $\delta_{m_{i}}=O\left(\frac{1}{\left[m_{i}\right]_{q_{i}}}\right)$ for $i=1,2$, which completes the proof of Theorem 10 .

\section{Conclusions}

In our investigation, we introduced the bivariate form of the previous operators [13] and then obtained the GBS-type approximation in the Bögel continuous function. These types of constructions of operators give an extended form of Jakimovski-Leviatan-Beta type $q$-integral operators given by [13]. We studied the bivariate properties of our new operators by the use of the modulus of continuity and the mixed-modulus of continuity and then obtained the degree of approximations for Lipschitz-class maximal functions. Moreover, we are mentioning here that the convergence at a certain point by GBS-type operators defined by (46) is more generalized than the bivariate operator (15) and the operator defined by [13]. Finally, in our future work, we will study the statistical approximation of these operators, as well as the approximation properties, by using power series methods, including some shape-preserving properties of these types operators.

Funding: This research received no external funding.

Institutional Review Board Statement: Not applicable.

Informed Consent Statement: Not applicable.

Data Availability Statement: Not Applicable.

Conflicts of Interest: The author declares no conflict of interest.

\section{References}

1. Szász, O. Generalization of Bernstein's polynomials to the infinite interval. J. Res. Nat. Bur. Stds. 1950, 45, 239-245. [CrossRef]

2. Appell, P. Une classe de polynômes. Ann. Sci. École Norm. Sup. 1880, 9, 119-144. [CrossRef]

3. Büyükyazıcı, İ. Tanberkan, H.; Serenbay, S.; Atakut, C. Approximation by Chlodowsky type Jakimovski-Leviatan operators. J. Comput. Appl. Math. 2014, 259, 153-163. [CrossRef]

4. Cai, Q.B.; Çekim, B.; Íçöz, G. Gamma Generalization Operators Involving Analytic Functions. Mathematics 2021, 9, 1547. [CrossRef]

5. Çekim, B.; Aktaş, R.; İçöz, G. Kantorovich-Stancu type operators including Boas-Buck type polynomials. Hacet. J. Math. Stat. 2019, 48, 460-471. [CrossRef]

6. Çekim, B.; Taşdelen, F. A Kantorovich-Stancu Type Generalization of Szasz Operators including Brenke Type Polynomials. J. Funct. Spaces Appl. 2013, 9, 935430.

7. Mursaleen, M.; Khan, T. On approximation by Stancu type Jakimovski-Leviatan-Durrmeyer operators. Azerb. J. Math. 2017, 7, 16-26. [CrossRef]

8. Sucu, S.; Varma, S. Approximation by Sequence of Operators Involving Analytic Functions. Mathematics 2019, 7, 188. [CrossRef]

9. Sucu, S.; Íçöz, G.; Varma, S. On Some Extensions of Szász Operators Including Boas-Buck-Type Polynomials. Abstr. Appl. Anal. 2012, 2012, 680340. [CrossRef]

10. Varmaa, S.; Sucua, S.; İçözb, G. Generalization of Szasz operators involving Brenke type polynomials. Comput. Math. Appl. 2012, 64, 121-127. [CrossRef]

11. Al-Salam, W.A. q-Appell polynomials. Ann. Mat. Pura Appl. 1967, 4, 31-45. [CrossRef]

12. Keleshteri, M.E.; Mahmudov, N.I. A study on q-Appell polynomials from determinantal point of view. Appl. Math. Comp. 2015, 260, 351-369. [CrossRef]

13. Alotaibi, A.; Mursaleen, M. Approximation of Jakimovski-Leviatan-Beta type integral operators via $q$-calculus. AIMS Math. 2020, 5, 3019-3034. [CrossRef]

14. Nasiruzzaman, M. Approximation properties by Szász operators to bivariate functions via Dunkl analogue. Iran. J. Sci. Technol. Trans. Sci. 2021, 45, 259-269. [CrossRef]

15. Nasiruzzaman, M.; Aljohani, A.F. Approximation by Szász-Jakimovski-Leviatan type operators via aid of Appell polynomials. J Funct. Spaces 2020, 2020, 9657489. [CrossRef]

16. Nasiruzzaman, M.; Aljohani, A.F. Approximation by parametric extension of Szász-Mirakjan-Kantorovich operators involving the Appell polynomials. J. Funct. Spaces 2020, 2020, 8863664. [CrossRef]

17. Nasiruzzaman, M.; Mursaleen, M. Approximation by Jakimovski-Leviatan-Beta operators in weighted space. Adv. Differ. Equ. 2020, 2020, 393. [CrossRef] 
18. Nasiruzzaman, M.; Rao, N.; Kumar, M.; Kumar, R. Approximation on bivariate parametric-extension of Baskakov-Durrmeyeroperators. Filomat 2021, 35, 2783-2800. [CrossRef]

19. Nasiruzzaman, M.; Ansari, K.J.; Mursaleen, M. On the parametric approximation results of Phillips operators involving the q-Appell polynomials. Iran. J. Sci. Technol. Trans. Sci. 2021, 46, 251-263. [CrossRef]

20. Mohiuddine, S.A.; Acar, T.; Alotaibi, A. Construction of a new family of Bernstein-Kantorovich operators. Math. Meth. Appl. Sci. 2017, 40, 7749-7759. [CrossRef]

21. Mohiuddine, S.A.; Ahmad, N.; Özger, F.; Alotaibi, A.; Hazarika, B. Approximation by the parametric generalization of BaskakovKantorovich operators linking with Stancu operators. Iran. J. Sci. Technol. Trans. Sci. 2021, 45, 593-605. [CrossRef]

22. Mohiuddine, S.A.; Hazarika, B.; Alghamdi, M.A. Ideal relatively uniform convergence with Korovkin and Voronovskaya types approximation theorems. Filomat 2019, 33, 4549-4560. [CrossRef]

23. Mohiuddine, S.A.; Özger, F. Approximation of functions by Stancu variant of Bernstein-Kantorovich operators based on shape parameter $\alpha$. Rev. R. Acad. Cienc. Exactas Fís. Nat. Ser. A Math. RACSAM 2020, 114, 70. [CrossRef]

24. Mursaleen, M.; Ansari, K.J.; Nasiruzzaman, M. Approximation by $q$-analogue of Jakimovski-Leviatan operators involving q-Appell polynomials. Iran. J. Sci. Technol. Trans. Sci. 2017, 41, 891-900. [CrossRef]

25. Mursaleen, M.; Nasiruzzaman, M. Dunkl generalization of Kantorovich type Szasz-Mirakjan operators via $q$-calculus. Asian Eur. J. Math. 2017, 10, 1750077. [CrossRef]

26. Acar, T.; Kajla, A. Degree of Approximation for bivariate generalized Bernstein type operators. Results Math. 2018, 73, 79. [CrossRef]

27. Kajla, A.; Mohiuddine, S.A.; Alotaibi, A. Blending-type approximation by Lupaş-Durrmeyer-type operators involving Pólya distribution. Math. Meth. Appl. Sci. 2021, 44, 9407-9418. [CrossRef]

28. Özger, F.; Srivastava, H.M.; Mohiuddine, S.A. Approximation of functions by a new class of generalized Bernstein-Schurer operators. Rev. R. Acad. Cienc. Exactas Fís. Nat. Ser. A Math. RACSAM 2020, 114, 173. [CrossRef]

29. Rao, N.; Nasiruzzaman, M.; Heshamuddin, M.; Shadab, M. Approximation properties by modified Baskakov-Durrmeyer operators based on shape Parameter $\alpha$. Iran. J. Sci. Technol. Trans. Sci. 2021, 45, 1457-1465. [CrossRef]

30. Rao, N.; Wafi, A.; Acu, A.M. q-Szász-Durrmeyer type operators based on Dunkl analogue. Complex Anal. Oper. Theory 2019, 13, 915-934. [CrossRef]

31. Gadẑiev, A.D. Positive linear operators in weighted spaces of functions of several variables, Izv. Akad. Nauk Azerbaidzhan. SSR Ser. Fiz.-Tekhn. Mat. Nauk. 1980, 4, 32-37.

32. Gadziev, A.D.; Hacisalihoglu, H. Convergence of the Sequences of Linear Positive Operators; Ankara University: Yenimahalle, UK, 1995.

33. Agrawal, P.N.; Ispir, N.; Kajla, A. GBS Operators of Lupaş-Durrmeyer Type Based on Polya Distribution. Results Math. 2016, 69, 397-418. [CrossRef]

34. Bărbosu, D. On the approximation some GBS type operators. Bul. Ştiinţ. Univ. Baia. Mare Ser. B Math.-Inform. 2000, 16, $247-254$.

35. Bögel, K. Mehrdimensionale differentiation von Funktionen mehrerer veränderlichen. J. Reine Angew. Math. 1934, 170, 197-217.

36. Bögel, K. Über die mehrdimensionale differentiation. Jahresber. Dtsch. Math. Ver. 1935, 65, 45-71.

37. Cai, Q.B.; Cheng, W.T.; Çekim, B. Bivariate $\alpha, q$-Bernstein-Kantorovich operators and GBS operators of bivariate $\alpha, q$-BernsteinKantorovich type. Mathematics 2019, 7, 1161. [CrossRef]

38. Mohiuddine, S.A. Approximation by bivariate generalized Bernstein-Schurer operators and associated GBS operators. Adv. Differ. Equ. 2020, 2020, 676. [CrossRef] 